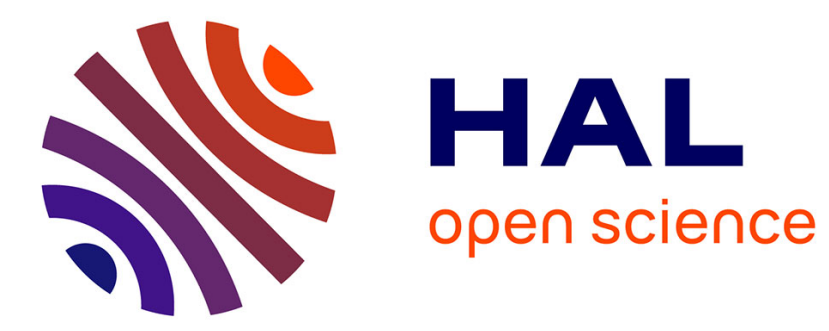

\title{
Multiple Tests based on a Gaussian Approximation of the Unitary Events method with delayed coincidence count
}

\author{
Christine Tuleau-Malot, Amel Rouis, Franck Grammont, Patricia \\ Reynaud-Bouret
}

\section{To cite this version:}

Christine Tuleau-Malot, Amel Rouis, Franck Grammont, Patricia Reynaud-Bouret. Multiple Tests based on a Gaussian Approximation of the Unitary Events method with delayed coincidence count. 2013. hal-00757323v2

\section{HAL Id: hal-00757323 \\ https://hal.science/hal-00757323v2}

Preprint submitted on 28 Jun 2013 (v2), last revised 21 Feb 2014 (v4)

HAL is a multi-disciplinary open access archive for the deposit and dissemination of scientific research documents, whether they are published or not. The documents may come from teaching and research institutions in France or abroad, or from public or private research centers.
L'archive ouverte pluridisciplinaire HAL, est destinée au dépôt et à la diffusion de documents scientifiques de niveau recherche, publiés ou non, émanant des établissements d'enseignement et de recherche français ou étrangers, des laboratoires publics ou privés. 


\section{Multiple Tests based on a Gaussian Approxima- tion of the Unitary Events method with delayed coincidence count}

\section{Christine Tuleau-Malot}

malot@unice.fr

Univ. Nice Sophia Antipolis, CNRS, LJAD, UMR 7351, 06100 Nice, France

\section{Amel Rouis}

rouis.a@chu-nice.fr

Centre Hospitalier Universitaire de Nice, France

\section{Franck Grammont ${ }^{1}$}

grammont@unice.fr

Univ. Nice Sophia Antipolis, CNRS, LJAD, UMR 7351, 06100 Nice, France

\section{Patricia Reynaud-Bouret}

reynaudb @unice.fr

Univ. Nice Sophia Antipolis, CNRS, LJAD, UMR 7351, 06100 Nice, France

Keywords: Unitary Events - Multiple Shift - Synchronization - Multiple testing - Independence tests - Poisson processes - Neuronal assemblies

The Unitary Events (UE) method is one of the most popular and efficient methods used this last decade to detect patterns of coincident joint spike activity among simultaneously recorded neurons. The detection of coincidences is usually based on binned coincidence count (Grün, 1996), which is known to be subject to information loss (Grün et al., 1999). This defect has been corrected by the multiple shift coincidence count (Grün et al., 1999). The statistical properties of this count have not been further investigated until the present work, the formula being more

\footnotetext{
${ }^{1}$ Corresponding author
} 
difficult to deal with than the original binned count. First of all, we propose a new notion of coincidence count, the delayed coincidence count which is equal to the multiple shift coincidence count when discretized point processes are involved as models for the spike trains. Moreover, it generalizes this notion to non discretized point processes, allowing us to propose a new Gaussian approximation of the count. Since unknown parameters are involved in the approximation, we perform a plug-in step, where unknown parameters are replaced by estimated ones, leading to a modification of the approximating distribution. Finally the method takes the multiplicity of the tests into account via a Benjamini and Hochberg approach (Benjamini \& Hochberg, 1995), to guarantee a prescribed control of the False Discovery Rate. We compare our new method, called MTGAUE for Multiple Tests based on a Gaussian Approximation of the Unitary Events, and the UE method proposed in (Grün et al., 1999) over various simulations, showing that MTGAUE extends the validity of the previous method. In particular, MTGAUE is able to detect both profusion and lack of coincidences with respect to the independence case and is robust to changes in the underlying model. Furthermore MTGAUE is applied on real data.

\section{Introduction}

The study of how neural networks transmit activity in the brain and somehow code information implies to consider various aspects of spike activity. Historically, firing rates have been firstly considered as the main way for neurons or populations of neurons to transmit activity, in correlation with some experimental or behavioral events. Such kinds of correlations have been shown mainly by the use of Peri-Stimulus-TimeHistograms (PSTH) (Abeles, 1982; Gerstein \& Perkel, 1969; Shinomoto, 2010).

However, beside the role of firing rate, it has been argued, theoretically at the beginning, that the activity of ensemble of neurons may be coordinated in the spatiotemporal domain (i.e. coordination of the production of spikes between different neurons) to form neuronal assemblies (Hebb, 1949; Palm, 1990; Sakurai, 1999; Von der Malsburg, 1981). Indeed, such assemblies could be constituted on the basis of specific spike timing, thanks to several mechanisms at the synaptic level (König et al., 1996; Rudolph \& Destexhe, 2003; Softky \& Koch, 1993). The required neural circuitry could spontaneously emerge with spike-timing-dependent plasticity (Brette, 2012). Such coordinated activity could easily propagate over neural networks (Abeles, 1991; Diesmann

et al., 1999; Goedeke \& Diesmann, 2008) and be used as a potential "code" for the brain (Singer, 1993, 1999). Moreover simulation studies have shown how synchronization emerges and propagates in neural networks, with or without oscillations (Diesmann 
et al., 1999; Golomb \& Hansel, 2000; Tiesinga \& Sejnowski, 2004; Goedeke \& Diesmann, 2008; Rudolph \& Destexhe, 2003).

In addition to these theoretical considerations0, many experimental evidences have been accumulated, which show that coordination between neurons is indeed taking place. In particular, it has been shown that the mechanisms of spike generation can be very precise (Mainen \& Sejnowski, 1995) under physiological conditions (Boucsein et al., 2011; Konishi et al., 1988; Lestienne, 2001; Prescott et al., 2008). Spike synchronization, with or without oscillations, has been shown to be involved in the so-called binding problem (Engel \& Singer, 2001; Singer \& Gray, 1995; Singer, 1999; Super et al., 2003). Spike synchronization has also been studied in relation with firing rate (Abeles \& Gat, 2001; Eyherabide et al., 2009; Gerstein, 2004; Grammont \& Riehle, 1999, 2003; Heinzle et al., 2007; König et al., 1996; Kumar et al., 2010; Lestienne, 1996; Maldonado et al., 2000; Masuda \& Aihara, 2007; Riehle et al., 1997; Vaadia et al., 1995).

Most of these experimental evidences could not have been obtained without the development of specific descriptive analysis methods of spike-timing over the last decades: cross-correlogram (Perkel et al., 1967), Gravitational Clustering (Gerstein \& Aertsen, 1985) or Joint-Peri-Stimulus-Time-Histogram (JPSTH) (Aertsen et al., 1989). However, these methods do not necessarily answer to a major criticism that considers that spike synchronization might just be an epiphenomenon of the variations of the firing rate. That is why, in direct line with these methods, Grün and collaborators developed the Unitary Events (UE) analysis method (Grün, 1996).

The UE method is originally based on a binned coincidence count (see also Section 2.1 for a more precise definition). This method has been continuously improved until today (Grün et al., 2001a,b; Grün, 2009; Grün et al., 2010; Gütig et al., 2001; Grün et al., 2003; Pipa \& Grün, 2003; Pipa et al., 2003; Louis et al., 2010; Pipa et al., 2013). It is a popular method which has been used successfully in several experimental studies (Riehle et al., 1997, 2000; Grammont \& Riehle, 1999, 2003; Maldonado et al., 2008). However, these approaches suffer from several defects due to the use of the binned coincidence count. Indeed, as pointed out in (Grün et al., 1999, 2010), there may be a large information loss, coincidences being discarded when they correspond to two spikes lying in two adjacent distinct bins. Up to $60 \%$ of the coincidences can be lost when the bin length is the typical delay/jitter between two spikes participating to the same coincidence. Another version of the UE method has consequently been proposed: the multiple shift coincidence count (Grün et al., 1999) (see Section 2.1 for a precise definition, see also (Pazienti, 2008) for another development). However, and up to our knowledge, this notion has not been as well explored as the notion of binned coincidence count. Indeed and as already pointed out in (Grün et al., 2010), the various shifts 
can make the coincidence count more complex than a sum of independent variables, depending on the underlying model.

Therefore the main aim of this article is to complete the study of this notion of multiple shift coincidence count and to propose a new method which extends the validity of the original multiple shift UE method (Grün et al., 1999). To do so, we generalize the notion, that was given for discretized spike trains at a certain resolution level. The delayed coincidence count defined in Section 2.3 is exactly the same coincidence count for discretized spike trains but this new formula can now be also applied to non discretized point processes as well. This mathematical notion allows us to compute the expectation and the variance in the simplest case of Poisson processes, which approximate Bernoulli processes used in (Grün et al., 1999). Therefore Fano's factor can be derived for the multiple shift / delayed coincidence count, as it has been done for instance in (Pipa et al., 2013) for the more classical notion of binned coincidence count. This also leads to a Gaussian approximation of the distribution of the multiple shift / delayed coincidence count, when Poisson or Bernoulli processes are involved as models for the spike trains.

However this approximation depends on unknown parameters in practice, namely the underlying firing rates. Such problems due to unknown parameters can be bypassed for the binned coincidence count either by trial-shuffling (Pipa \& Grün, 2003; Pipa et al., 2003 ) - with some non trivial and specific implementation solutions - or by conditioning on the observed number of spikes (Gütig et al., 2001) - the solution being completely linked to the form of the binned coincidence count and the use of Bernoulli models. Both solutions are consequently not used here for the multiple shift coincidence count, which is more intricate than classical binned coincidence count. We prefer to look more carefully at the replacement of the unknown firing rates by estimated ones, step which is known in statistics as a plug-in step. By looking closely at the plug-in procedure, we show in Section 3.1 that it changes the variance of the asymptotic Gaussian distribution, and, therefore, we correct the approximation to take this phenomenon into account. Up to our knowledge, no correction due to the plug-in effect has been taken into account even for the classical binned coincidence count. The last step (Section 3.2) of our procedure consists in carefully controlling the False Discovery Rate (FDR), when several windows of analysis are considered, by using Benjamini and Hochberg procedure (Benjamini \& Hochberg, 1995).

Each time a thorough simulation study shows the actual performance of our procedure. A real data analysis is also performed in Section 4 on data that have already been partially published, so that the detection ability of the method can be tested in concrete situations. Finally, we discuss the overall improvements due to our procedure with respect to the original method of (Grün et al., 1999) in Section 5. 
In all the sequel, we write in italic technical expressions, the first time they are encountered and we give in the same paragraph their definition. We also use the following notation, that are the ones generally used in point process theory (Daley \& Vere-Jones, 2003). A point process $N$ is a random countable set of points (of $\mathbb{R}_{+}$here). Each point corresponds to the detection time of a spike of the considered neuron by the recording electrode. For any set $A$ of $\mathbb{R}_{+}, N(A)$ is the number of points in $A$ and $N(d t)$ is the associated point measure, that is $N(d t)=\sum_{S \in N} \delta_{S}$ where $\delta_{S}$ is the Dirac measure at the point $S$. This means that for every function $f, \int f(t) N(d t)=\sum_{S \in N} f(S)$. The point process corresponding to the spike train of neuron $j$ is denoted $N_{j}$ and when $M$ trials are recorded, the point process corresponding to the spike train of neuron $j$ during trial $m$ is denoted $N_{j}^{(m)}$. In all the sequel and whatever the chosen model, we assume that the $M$ trials are independent and identically distributed (i.i.d.), which means ergodicity across the trials, except when precisely stated otherwise. We denote by $\mathbb{P}$ the probability measure, by $\mathbb{E}$ its corresponding expectation and by Var its corresponding variance. Also $\mathbf{1}_{A}$ denotes the indicator function of the event $A$, which takes value 1 when $A$ is true and 0 otherwise.

Fundamental notions for the present article are given in the following definition (see also (Staude et al., 2010) for this kind of distinction).

Definition 1: Real single unit data are recorded with a certain resolution $h$, which is usually $10^{-3} \mathrm{~s}$ or $10^{-4} \mathrm{~s}$ depending on the experiment. Formally time is cut into intervals of length $h$ and of the form $[i h-h / 2, i h+h / 2)$. Then one associates to any point process, $N$, its associated sequence at resolution $h$, i.e. a sequence of 0 and $1,\left(H_{n}\right)_{n}$, where $H_{i}=1$ corresponds to the presence of (at least) one point of $N$ in $[i h-h / 2, i h+h / 2)$. Reciprocally, to a sequence of 0 and $1,\left(H_{n}\right)_{n}$, we associate a point process $N$ by taking the set of all points of the type $S=i h$ such that $H_{i}=1$. Such point process that is forced to have only points of the type $i h$, for some integer $i$, is called discretized at resolution $h$.

\section{Probabilistic study of the coincidence count}

\subsection{The multiple shift coincidence count}

A pair of neurons being recorded, the question is how to properly define a coincidence and more precisely, the coincidence count. Usually those counts are computed over several windows of time. We focus in this section on only one window $W$ of length $T$.

In classical UE methods, both spike trains are usually represented by sequences of 0 and 1 of length $r=T /(d \times h)$, for some integer $d \geq 1$ (Grün, 1996; Grün et al., 2010). The presence of 1 at position $i$ indicates that there is at least one spike in the $i$ th segment 
of length $d \times h$. The segments of length $d \times h$ are usually called bins and are centred around points of the type $i d \times h$. The previous construction means that the real data have been binned at a coarser level (namely $d \times h$ ) than their original resolution $h$. We denote by $D_{1}^{1}, \ldots, D_{r}^{1}$ and by $D_{1}^{2}, \ldots, D_{r}^{2}$, the associated sequence to the first and second neuron respectively. According to this bin construction, a coincidence at time $C=i d \times h$ happens if $D_{i}^{1}=D_{i}^{2}=1$. The coincidence count, in this binning framework, is then the number of $i$ 's such that $D_{i}^{1}=D_{i}^{2}=1$. The problem, underlined in (Grün et al., 1999), is that for reasonable $d$ (typically $d \times h=5 \cdot 10^{-3}$ s), a significant number of spikes that are at distance less than $d \times h$ are not counted if they fall in two adjacent and distinct bins: the binning effect generates a significative loss of information.

The multiple shift method, introduced in (Grün et al., 1999), uses a very particular notion of coincidence count that corrects this information loss and which can be described as follows (see Figure 6 of (Grün et al., 1999)). The spike trains are kept at their original resolution level $h$. Both spike trains, observed on a window $W$ at resolution $h$, are still considered as a sequence of 0 and 1 , denoted $H_{1}^{1}, \ldots, H_{n}^{1}$ and $H_{1}^{2}, \ldots, H_{n}^{2}$ respectively in the sequel. Then a (near) coincidence is observed at time ih on the window $W$, if there exists a shift $j$, integer in $\{-d, \ldots, d\}$, such that $H_{i}^{1}=H_{i+j}^{2}=1$. Note that this definition implies in particular that such a $j$ should also satisfy that

$$
1 \leq i+j \leq n
$$

as clearly presented in Figure 6 of (Grün et al., 1999), i.e. that the recordings outside the window $W$ of interest are discarded. The multiple shift coincidence count is then defined by the total number of (near) coincidences on the window $W$, i.e. formally speaking

$$
X=\sum_{i=1}^{n} \sum_{j /|j| \leq d} \mathbf{1}_{H_{i}^{1}=H_{i+j}^{2}=1} .
$$

Taking $k=i+j$ in the previous sum, we obtain

$$
X=\sum_{i=1}^{n} \sum_{k=1}^{n} \mathbf{1}_{|k-i| \leq d} \mathbf{1}_{H_{i}^{1}=1} \mathbf{1}_{H_{k}^{2}=1} .
$$

Hence, it can be also understood as the total number of couples $(i, k)$ such that $H_{i}^{1}=$ $H_{k}^{2}=1$, such that $|k-i| \leq d$ and such that both $i$ and $k$ belong to $\{1, \ldots, n\}$. This present notion is therefore symmetric in the first and second spike trains.

If the condition (1) on $j$ is not fulfilled, and if we authorized a shift $j$, such that the corresponding time $(i+j) h$ does not necessarily belong to the window of interest $W$, then we are comparing in (3), a sequence of length $n$ for the first spike train and a sequence of length $n+2 d$ for the second spike train. Therefore the coincidence count would not be symmetric anymore. As a matter of fact, with $h=10^{-3}$ s, it is quite 
usual to have $n=100$ and up to $d=20$. Forgetting (1) may therefore lead to a huge asymmetry, one sequence being $40 \%$ larger than the other one.

All coincidence counts are classically used to detect dependence. They are compared to the distribution expected under the independence hypothesis. Usually, model of independence consequently need to be imposed and in many articles on the UE method (in particular in (Grün et al., 1999)), the spike trains are modelled by independent Bernoulli processes. More precisely, the $H_{i}^{j}$ are assumed to be independent and identically distributed Bernoulli variables with parameter $p_{j}=\lambda_{j} h$, where $\lambda_{j}$ is the firing rate of the neuron $j$.

In (Grün et al., 1999), the computation, based on the fact that $U_{i k}=\mathbf{1}_{H_{i}^{1}=1} \mathbf{1}_{H_{k}^{2}=1}$ is also a Bernoulli variable of parameter $p_{1} p_{2}$, states that there is approximately $(2 d+1) n$ such Bernoulli variables. Consequently, it was considered that the expected coincidence count is (see (15) of Grün et al. (1999))

$$
m_{g}=\lambda_{1} \lambda_{2} h^{2} n(2 d+1)
$$

The main problem with such a derivation is that when $i$ is close to 1 or $n$ one cannot always find $(2 d+1)$ indices $k$ such that $|k-i| \leq d$ and $k \in\{1, \ldots, n\}$. There could be much less. This edge effect is negligible for small $d$ but becomes more crucial when $d$ is large. We correct this effect in this article to extend the validity of the approximation developed in this article.

The second main problem is that if it is true that the present coincidence count is a sum of Bernoulli variables, it is not true that those variables are independent because the variable $H_{i}^{j}$ may participate in more than one coincidence. Therefore the present multiple shift coincidence count is not a Binomial variable when Bernoulli processes are considered. This fact makes the behavior of this precise multiple shift coincidence count different from other classical notions of coincidence count based on binning. Therefore the present work proposes a limit distribution for the coincidence count that takes this dependency into account, so that the approximation is valid for a larger set of parameters than the Poisson approximation done in (Grün et al., 1999). If it is quite difficult to directly do so for Bernoulli processes, this probabilistic result can be easily derived if we approximate Bernoulli processes by Poisson processes.

\subsection{Bernoulli and Poisson processes}

Recall that Bernoulli processes are generated as follows. For a window $W$ of length $T$, at the resolution $h, n=T / h$ independent Bernoulli variables, $B_{i}$, with parameter $p=\lambda h$ are simulated, where $\lambda$ is the firing rate of the considered neuron. The associated point process (see Definition 1) is denoted $N_{B}$ in the sequel. 
It is well known that when $h$ tends to 0 , then the Bernoulli process tends to a Poisson process. This can for instance be seen because the number of points, $N_{B}(W)$, is a binomial variable that tends in distribution towards a Poisson variable with parameter $\lambda T$ when $h$ tends to 0 . In particular the approximation is valid as soon as $n \geq 100$ and $p \leq 0.1$, (Hogg \& Tanis, 2009, p 159). Since, by construction, for any disjoint sets $A_{1}, \ldots, A_{k}, N_{B}\left(A_{1}\right), \ldots, N_{B}\left(A_{k}\right)$ are independent variables, we recover the classical definition of a homogeneous Poisson process of intensity $\lambda$ (see (Daley \& Vere-Jones, 2003) for a precise definition). Note that Poisson processes are not discretized at any resolution level, whereas Bernoulli processes are (see Definition 1).

More precisely, in our set-up, windows of length $0.1 \mathrm{~s}$ are classically considered, with firing rates less than $100 \mathrm{~Hz}$ and with resolution $h=10^{-3} \mathrm{~s}$ or $h=10^{-4} \mathrm{~s}$. We are consequently typically in parameters region where the Poisson approximation is valid. In (Reynaud-Bouret et al., 2013), several classical tests, originally due to (Ogata, 1988), have been proposed to test whether a point process is a homogeneous Poisson process or not and we refer the reader to this article for detailed explanations on the procedures. In Figure 1, we run those tests on a simulated Bernoulli process. The p-values ${ }^{2}$ are large, meaning that the various tests accept the Poisson assumption (see also (Ventura, 2010) for precise definitions of tests and p-values). Moreover the repartitions of the $\mathrm{p}$-values are close to the diagonal meaning that the distributions of the various statistics (positions, numbers of the spikes or delays between spikes) are the ones given by a classical Poisson process, and they become closer to the diagonal when $h$ decreases.

Hence, Bernoulli processes can be well approximated by Poisson processes on the typical set of parameters used in neuroscience and they are even statistically indistinguishable from Poisson processes, at the resolution $h=10^{-4}$ s.

\subsection{The delayed coincidence count}

If we want to use Poisson processes instead of Bernoulli processes to perform the computations, we need to rewrite the multiple shift coincidence count in terms of point processes that are not necessarily discretized at the resolution level $h$ (see Definition 1$)$. To turn (3) into a more generic formula valid for any point process, let us remark the following phenomenon. Fix $i \leq k$ and fix some $x=i h$ as point of $N_{1}$. If $N_{2}$ is not discretized and if we consider its associated sequence $\left(H_{1}^{2}, \ldots, H_{n}^{2}\right)$ at resolution $h$ (see

\footnotetext{
2 A p-value is the random value of $\alpha$ for which a test of level $\alpha$ passes from "accept" to "reject". Note that usually when $\alpha=0$, the test always accepts, whereas it always rejects when $\alpha=1$ : therefore there is a limit value which depends on the observations for which one passes from one decision to another one. If the test is of Type I error exactly $\alpha$ for all $\alpha$, then one can prove that the corresponding p-value is uniformly distributed on $[0,1]$ under $H_{0}$. Therefore their value as position of their normalized rank over a certain i.i.d. sample should be close to the diagonal of the square.
} 


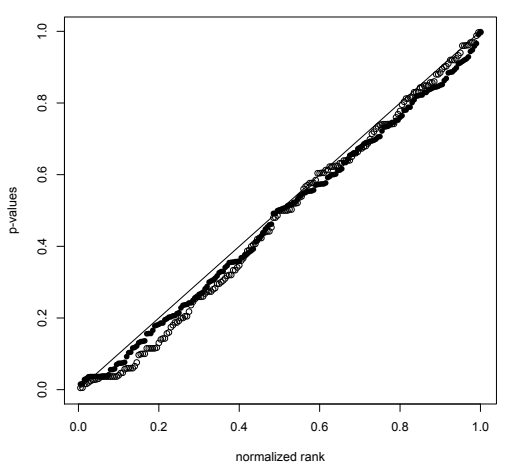

A

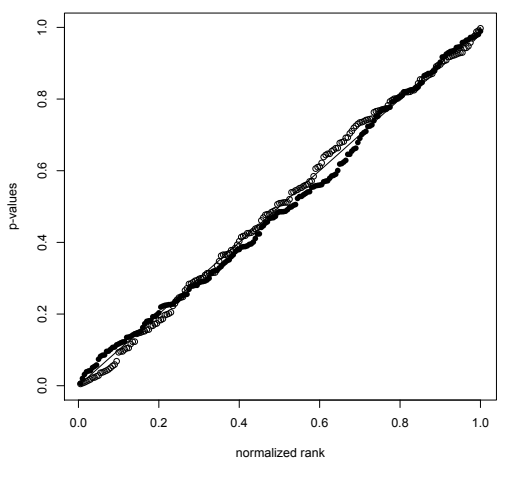

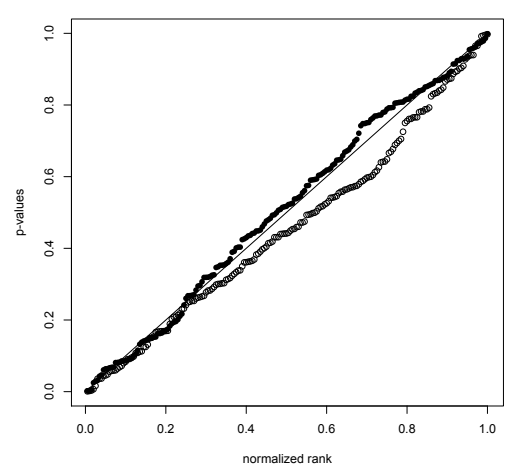

B

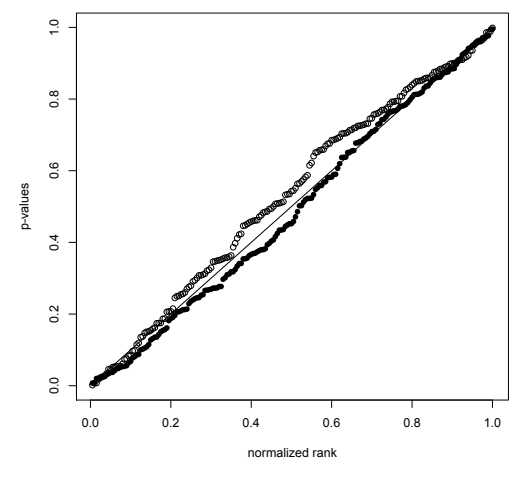

Figure 1: Poisson approximation of the Bernoulli process. 200 p-values for the following tests are displayed as function of their rank divided by 200 . The diagonal is represented by a black line. The firing rate of the Bernoulli process is $\lambda=30 \mathrm{~Hz}$ and the p-values corresponding to $h=10^{-3}$ s are represented by an empty circle, whereas the p-values corresponding to $h=10^{-4}$ s are represented by a black point. In $\mathbf{A}$, the process is simulated 200 times on a window of length $T=0.1$ s and each time a KolmogorovSmirnov uniformity test is performed. In $\mathbf{B}$, the aggregated version over 100 simulated trials of the Kolmogorov-Smirnov uniformity test is performed 200 times, with $T=0.1$ s. In $\mathbf{C}$, the chi-square Poisson test over 100 trials is performed 200 times, with $T=0.1$ s. In $\mathbf{D}$, the exponentiality test of the delays between points over only 1 trial of length 10 s is performed 200 times.

Definition 1), then a point $y$ of $N_{2}$ that corresponds to a $k$ such that $|k-i| \leq d$, could be as far as $d \times h+h / 2$ and still counted as a near coincidence. In particular, when $d=0$, $y$ is in the segment of center $x$ with length $h$ if and only if $|y-x| \leq h / 2$. Therefore, let $\delta=d \times h+h / 2$. The delayed coincidence count, generalizing the notion of multiple shift coincidence count for general point process, can be written as

$$
X=\int_{W^{2}} \mathbf{1}_{|x-y| \leq \delta} N_{1}(d x) N_{2}(d y) .
$$


When $N_{1}$ and $N_{2}$ are discretized with resolution $h$, both definitions (2) and (5) coincide and both coincidence counts are exactly the same.

Now let us translate the multiple shift method introduced by Grün and her collaborators to the general point process framework. Equation (4) can be easily rewritten as

$$
m_{g}=2 \delta T \lambda_{1} \lambda_{2}
$$

where $T$ is the length of the window $W$, and it is used that the distribution of $X$ may be approximated by a Poisson distribution with parameter $m_{g}$ (see (Grün et al., 1999)).

Here, assuming that both $N_{1}$ and $N_{2}$ are now Poisson processes, one can prove the following result.

Theorem 1. Let us fix $\delta$ in (5) such that

$$
0<2 \delta<T
$$

where $T$ is the length of the window $W$. If $N_{1}$ and $N_{2}$ are independent homogeneous Poisson processes with respective intensity $\lambda_{1}$ and $\lambda_{2}$, then the expectation of the delayed coincidence count $X$ and its variance are given by

$$
m_{0}:=\mathbb{E}(X)=\lambda_{1} \lambda_{2}\left[2 \delta T-\delta^{2}\right]
$$

and

$$
\sigma^{2}:=\operatorname{Var}(X)=\lambda_{1} \lambda_{2}\left[2 \delta T-\delta^{2}\right]+\left[\lambda_{1}^{2} \lambda_{2}+\lambda_{1} \lambda_{2}^{2}\right]\left[4 \delta^{2} T-\frac{10}{3} \delta^{3}\right] .
$$

Moreover if $M$ i.i.d trials are available, then

$$
\sqrt{M} \frac{\bar{m}-m_{0}}{\sqrt{\sigma^{2}}} \stackrel{\mathcal{L}}{\rightarrow} \mathcal{N}(0,1)
$$

where $\bar{m}$ is the average observed coincidence count with delay $\delta$, i.e.

$$
\bar{m}=\frac{1}{M} \sum_{m=1}^{M} X^{(m)} \quad \text { with } \quad X^{(m)}=\int_{W^{2}} \mathbf{1}_{|x-y| \leq \delta} N_{1}^{(m)}(d x) N_{2}^{(m)}(d y) .
$$

The symbol $\stackrel{\mathcal{L}}{\rightarrow}$ means convergence in distribution when $M$ tends to infinity. This means for instance that the quantiles of $\sqrt{M / \sigma^{2}}\left(\bar{m}-m_{0}\right)$ tend to those of $\mathcal{N}(0,1)$, when $M$ becomes larger.

This result states first that $\mathbb{E}(X)$ can be computed when both observed point processes are independent homogeneous Poisson processes and that the edge effects appear in $m_{0}$ via a quadratic term in $\delta$ which is the difference with respect to $m_{g}$. Therefore it needs to be taken into account if one wants to compute delayed coincidence count with 
large $\delta$. Note that if (7) is not satisfied, then all the couples $(x, y)$ in $W$ are affected by this edge effect and in this case, the above formula for the expectation and variance are not valid anymore.

Note also that the Fano factor $^{3}$ i.e.

$$
F:=\frac{\operatorname{Var}(X)}{\mathbb{E}(X)}=1+2\left(\lambda_{1}+\lambda_{2}\right) \delta(1+o(1)),
$$

is strictly larger than 1 . The gap between the variable $X$ and a Poisson variable increases with the firing rate and with $\delta$. Several papers have also considered the Fano factor for binned coincidence count showing that the distribution may be different from a Poisson distribution, but up to our knowledge nothing has been done for the multiple shift coincidence count. In particular, in the recent (Pipa et al., 2013), Fano factors for renewal processes are computed, only in the case of binned coincidence count. Multiple shift coincidence count and binned coincidence count are exactly the same when the size of the bin is $h$ for the binned coincidence count and when $d=0$ in (2) for the multiple shift coincidence count. Since delayed coincidence count is a generalization of multiple shift coincidence count, it is logical that we recover results of the same flavour as the ones of (Pipa et al., 2013), in the Poisson case, with $\delta=h / 2$. Note however that both results are not equivalent since they are not based on the same notion of coincidence count.

Using Poisson processes instead of Bernoulli processes allows us to produce such result for the generalization of multiple shift coincidence count to the more general not necessarily discretized point process case. In addition, let us conclude this section with a small simulation to underline the fact that the present approximation of (10) is not valid only for Poisson processes in theory but also for Bernoulli processes in practice.

On Figure 2, we can see that the three distributions, i.e. the one of the delayed coincidence counts for Poisson processes with $\delta=d \times h$, the one with $\delta=d \times h+$ $h / 2$ and the one of the multiple shift coincidence counts for Bernoulli processes with resolution $h$ are almost indistinguishable. They are all three very well approximated by the Gaussian approximation of (10) and the distinction between $\delta=d \times h$ or $\delta=d \times$ $h+h / 2$ cannot be made when $h=10^{-4}$ s. On the contrary, all the Poisson distributions either with mean $M m_{g}$ (biased with neglected edge effects) or $M m_{0}$ (unbiased with edge effects taken into account), with $\delta=d \times h$ or $\delta=d \times h+h / 2$ are not fitting the coincidence count distribution. There is in particular a clear problem in the mean itself, which is due to edge effects when $\delta$ is large (see Figures 2.A and 2.B). But even for small $\delta$ (see Figures 2.C and 2.D), if the mean seems more correct (the edge effects being negligible), the variance is too small.

As a summary of this section, note consequently that

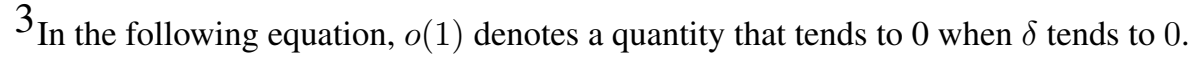




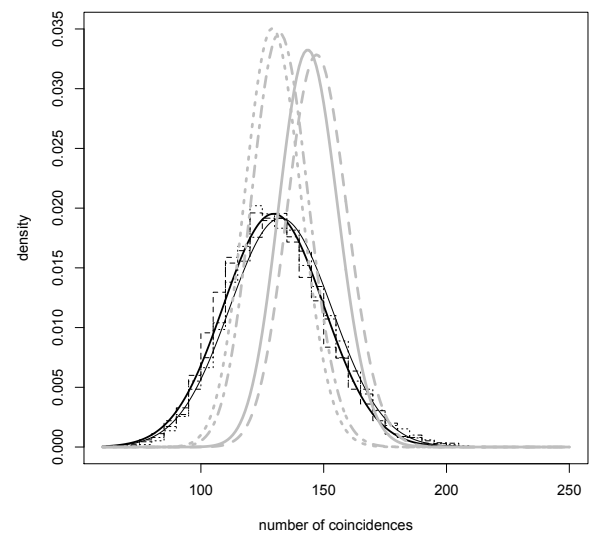

A

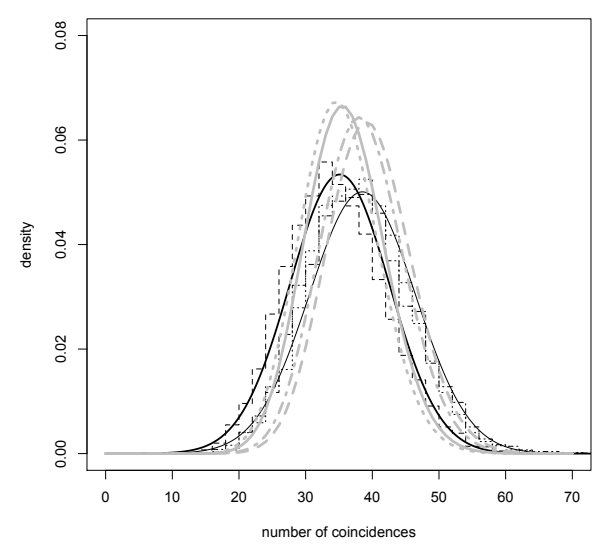

C

Figure 2: Repartition of the total coincidence count (i.e. the sum of the coincidence counts over $M$ trials). In all the experiments, $\lambda=30 \mathrm{~Hz}$ and $M=40$ trials are used. In dashed black (--), the empirical histogram over 5000 runs of the delayed coincidence counts with delay $\delta=x$, the Poisson processes being simulated. In dotdashed black $(\cdot-)$, same thing with $\delta=x+h / 2$. In dotted black $(\cdots)$, empirical histogram over 5000 runs of the multiple shift coincidence counts with $d$ such that $d \times h=x$, Bernoulli processes with resolution $h$ being simulated. In thick black, the density of a Gaussian variable with mean $M m_{0}$ and variance $M \sigma^{2}$, with $\delta=x$. In thin black, same thing with $\delta=x+h / 2$. In thick gray, the probability distribution function of a Poisson variable with mean $M m_{g}$ with $\delta=x$. In dashed gray, same thing with $\delta=x+h / 2$. In dotted gray, the probability distribution function of a Poisson variable with mean $M m_{0}$ with $\delta=x$. In dotdashed gray, same thing with $\delta=x+h / 2$. A corresponds to $h=10^{-3} \mathrm{~s}$ and $x=0.02 \mathrm{~s}, \mathbf{B}$ to $h=10^{-4} \mathrm{~s}$ and $x=0.02 \mathrm{~s}, \mathbf{C}$ corresponds to $h=10^{-3} \mathrm{~s}$ and $x=0.005 \mathrm{~s}$ and $\mathbf{D}$ to $h=10^{-4} \mathrm{~s}$ and $x=0.005 \mathrm{~s}$. 
- It is equivalent to simulate Poisson or Bernoulli processes for coincidence counts.

- Multiple shift coincidence counts are distributed as delayed coincidence counts, the latter being just the generalization of the former to the general point process theory.

- In both cases (Poisson or Bernoulli processes), the Gaussian approximation of (10) is valid, on the classical set of parameters.

- Edge effects need to be taken into account for large $\delta$.

- Considering $\delta=d \times h$ or $\delta=d \times h+h / 2$ with $h=10^{-4}$ s is completely equivalent.

Therefore, in the sequel, we use delayed coincidence count with $\delta$ of the type $d \times h$. Bernoulli processes are replaced by Poisson processes when necessary. When real data are considered, we use the resolution $h=10^{-4}$ s, which is the machine resolution of the recorded spike trains.

\section{Statistical study of the independence tests}

The previous section gives a probability result, namely the Gaussian approximation. Now let us see how this approximation can be turned into a fully operational statistical method. There are two main points that need to be taken into account. First, we do not know the value of $m_{0}$ in practice and we therefore need to plug an estimate in: how does this plug-in affect the distribution? Secondly, we usually consider several windows, therefore several tests are performed at once: how can one guarantee a small proportion of false positive for all the tests at once?

\subsection{Plug-in and modification of the Gaussian approximation}

In (10), neither $m_{0}$ nor $\sigma$ are known. Hence to perform the approximation in practice, we need to replace them by corresponding estimates, based on the observations. This step is known in statistics as a plug-in step and it is known to sometimes dramatically modify the distribution. One of the most famous example is the Gaussian distribution which has to be replaced by a Student distribution when the variance is unknown and estimated by an empirical mean over less than 30 realizations ${ }^{4}$ (Hogg \& Tanis, 2009, Table VI, p 658).

\footnotetext{
${ }^{4}$ More precisely, if $X_{1}, \ldots, X_{n}$ are i.i.d. Gaussian variables with mean $m$ and variance $\sigma^{2}$ then $\sqrt{n / \sigma^{2}} \sum_{i=1}^{n}\left(X_{i}-m\right) \sim \mathcal{N}(0,1)$ whereas $\sqrt{n / \hat{\sigma}^{2}} \sum_{i=1}^{n}\left(X_{i}-m\right) \sim T(n-1)$ where $\hat{\sigma}^{2}$ is the unbiased estimate of $\sigma^{2}$.
} 


\section{Theoretical study}

In the present set-up, as far as asymptotic in the number of trials $M$ is concerned, the plug-in of an estimate of $\sigma$ does not change the Gaussian distribution, whereas the plugin of an estimate of $m_{0}$ changes the variance of the limit, as we can see in the following result.

Theorem 2. With the same notation as in Theorem 1, let $\hat{\lambda}_{j}$ be the unbiased estimate of $\lambda_{j}$, the firing rate of neuron $j$, defined by

$$
\hat{\lambda}_{j}:=\frac{1}{M T} \sum_{m=1}^{M} N_{j}^{(m)}(W) .
$$

Let also $\hat{m}_{0}$ be an estimate of $m_{0}$ defined by

$$
\hat{m}_{0}:=\hat{\lambda}_{1} \hat{\lambda}_{2}\left[2 \delta T-\delta^{2}\right]
$$

Then under the assumptions of Theorem 1,

$$
\sqrt{M}\left(\bar{m}-\hat{m}_{0}\right) \stackrel{\mathcal{L}}{\rightarrow} \mathcal{N}(0, v),
$$

where

$$
v:=\lambda_{1} \lambda_{2}\left[2 \delta T-\delta^{2}\right]+\lambda_{1} \lambda_{2}\left[\lambda_{1}+\lambda_{2}\right]\left[\frac{2}{3} \delta^{3}-T^{-1} \delta^{4}\right] .
$$

Moreover $v$ can be estimated by

$$
\hat{v}:=\hat{\lambda}_{1} \hat{\lambda}_{2}\left[2 \delta T-\delta^{2}\right]+\hat{\lambda}_{1} \hat{\lambda}_{2}\left[\hat{\lambda}_{1}+\hat{\lambda}_{2}\right]\left[\frac{2}{3} \delta^{3}-T^{-1} \delta^{4}\right]
$$

and

$$
\sqrt{M} \frac{\bar{m}-\hat{m}_{0}}{\sqrt{\hat{v}}} \stackrel{\mathcal{L}}{\rightarrow} \mathcal{N}(0,1) .
$$

Figure 3 illustrates the impact of the plug-in in the renormalized coincidence count distribution.

When using plug-in, we need to renormalize the count since at each run a new value for the estimate is drawn. Therefore our reference in Figure 3 is the standard Gaussian variable. First we see that the Gaussian approximation of (10) is still valid, but more importantly that the plug-in steps of (15) and (18) are valid on Figure 3A. Instead of the new variance $v$ and its estimate $\hat{v}$, we have also plugged $\hat{m}_{0}$ in with $\sigma$ or a basic estimate of $\sigma$, namely

$$
\hat{\sigma}^{2}=\hat{\lambda}_{1} \hat{\lambda}_{2}\left[2 \delta T-\delta^{2}\right]+\left[\hat{\lambda}_{1}^{2} \hat{\lambda}_{2}+\hat{\lambda}_{1} \hat{\lambda}_{2}^{2}\right]\left[4 \delta^{2} T-\frac{10}{3} \delta^{3}\right] .
$$

The result in Figure 3B shows clearly that the variance $\sigma^{2}$ or the plug-in $\hat{\sigma}^{2}$ are wrong. Hence the plug-in correction definitely needs to be taken into account. Figures $\mathbf{3 C}$ and 


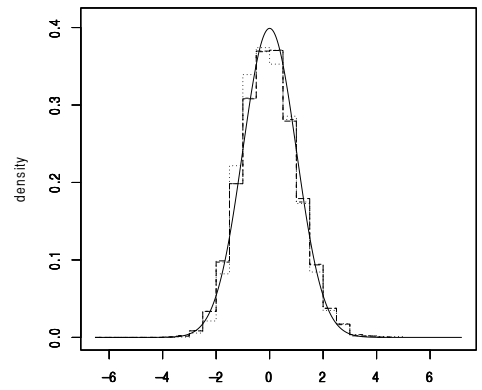

A

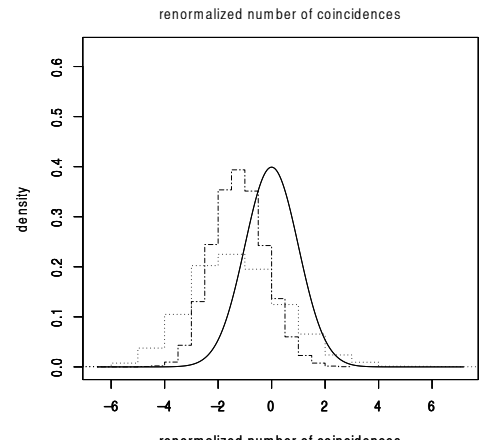

C

D

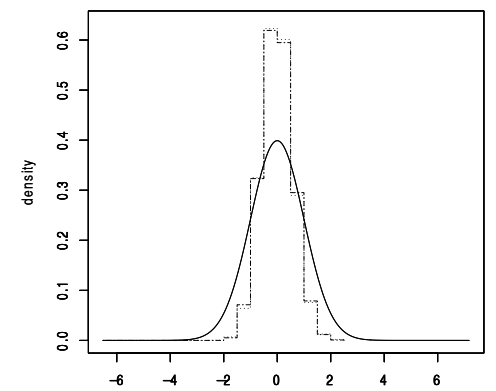

B

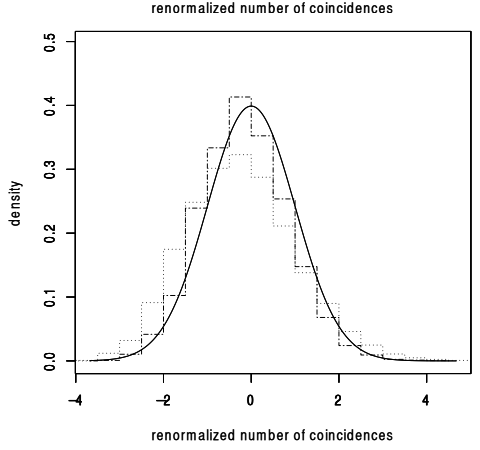

Figure 3: Repartition of the different renormalizations of the delayed coincidence count. Each time 5000 simulations of two independent Poisson processes with $\lambda_{1}=\lambda_{2}=$ $30 \mathrm{~Hz}$ and $M=40$ trials are performed. In all the graphs, the black thick curve is the density of a standard Gaussian variable $\mathcal{N}(0,1)$. In A, histograms of $\sqrt{M}\left(\bar{m}-m_{0}\right) / \sigma$ in dotted black $(\cdots)$, of $\sqrt{M}\left(\bar{m}-\hat{m}_{0}\right) / \sqrt{v}$ in dotdashed black (--), of $\sqrt{M}(\bar{m}-$ $\left.\hat{m}_{0}\right) / \sqrt{\hat{v}}$ in dashed black $(--)$, for $\delta=0.02$ s. In $\mathbf{B}$, histogram of $\sqrt{M}\left(\bar{m}-\hat{m}_{0}\right) / \sigma$ in dotted black $(\cdots)$, and histogram of $\sqrt{M}\left(\bar{m}-\hat{m}_{0}\right) / \hat{\sigma}$ in dashed black (--), for $\delta=0.02$ s. In $\mathbf{C}$, histogram of $\sqrt{M}\left(\bar{m}-m_{g}\right) / \sqrt{m_{g}}$ in dotted black $(\cdots)$ and histogram of $\sqrt{M}\left(\bar{m}-\hat{m}_{g}\right) / \sqrt{\hat{m}_{g}}$ in dotdashed black $(\cdot-)$, for $\delta=0.02$ s. In $\mathbf{D}$, same thing as in C but with $\delta=0.005$ s.

3D show what happens for the Poisson approximation of (Grün et al., 1999). More precisely, Poisson variables with parameter $\theta$ are well approximated by $\mathcal{N}(\theta, \theta)$, as soon as $\theta$ is large enough. So the variables are accordingly renormalized so that they can be plotted in the same space as the standard Gaussian variables. For such a large $\delta$ (here $\delta=0.02 \mathrm{~s}$ ), the Poisson approximation with parameter $M m_{g}$ or its estimation $M \hat{m}_{g}$ with

$$
\hat{m}_{g}=2 \hat{\lambda}_{1} \hat{\lambda}_{2} \delta T
$$

are not satisfactory either. Note however that $v$ in (16) is closer to $m_{0}$ than $\sigma^{2}$, for small $\delta$ since the corrective term is cubic. Therefore, for small $\delta$ (see Figure 3.D), the difference between our Gaussian approximation and the Poisson approximation becomes more negligible.

The Gaussian Approximation of the UE method - denoted GAUE in the following - 
given by Theorem 2, leads to three different single tests depending on what needs to be detected.

\section{Definition 2: The GAUE tests}

- the symmetric test $\Delta_{G A U E}^{s y m}(\alpha)$ of $H_{0}$ : " $N_{1}$ and $N_{2}$ are independent" versus $H_{1}$ : " $N_{1}$ and $N_{2}$ are dependent", which rejects $H_{0}$ when $\bar{m}$ and $\hat{m}_{0}$ are too different:

$$
\left|\bar{m}-\hat{m}_{0}\right| \geq z_{1-\alpha / 2} \sqrt{\frac{\hat{v}}{M}}
$$

- the unilateral test by upper value $\Delta_{G A U E}^{+}(\alpha)$ which rejects $H_{0}$ when $\bar{m}$ is too large:

$$
\bar{m} \geq \hat{m}_{0}+z_{1-\alpha} \sqrt{\frac{\hat{v}}{M}}
$$

- the unilateral test by lower value $\Delta_{G A U E}^{-}(\alpha)$ which rejects $H_{0}$ when $\bar{m}$ is too small:

$$
\bar{m} \leq \hat{m}_{0}-z_{1-\alpha} \sqrt{\frac{\hat{v}}{M}}
$$

where $z_{t}$ is the $t$-quantile of $\mathcal{N}(0,1)$, i.e. the real number $z_{t}$ such that $\mathbb{P}(\mathcal{N}(0,1) \leq$ $\left.z_{t}\right)=t$.

By Theorem 2, those three tests are asymptotically of Type I error $\alpha$, if the processes $N_{j}$ are homogeneous Poisson processes. It means that under this assumption, the probability that the test rejects the independence hypothesis, whereas the processes are independent, tends to $\alpha$ when the number of trials $M$ tends to infinity.

The original UE multiple shift method of (Grün et al., 1999) can be formalized in the same way.

$$
\begin{aligned}
& \text { Definition 3: The UE tests } \\
& \text { - a symmetric test } \Delta_{U E}^{s y m}(\alpha) \text { which rejects } H_{0} \text { when } M \bar{m} \leq q_{\alpha / 2} \text { or } M \bar{m} \geq \\
& q_{1-\alpha / 2} \\
& \text { - the unilateral test by upper value } \Delta_{U E}^{+}(\alpha) \text { which rejects } H_{0} \text { when } M \bar{m} \geq \\
& \quad q_{1-\alpha} \\
& \text { - the unilateral test by lower value } \Delta_{U E}^{-}(\alpha) \text { which rejects } H_{0} \text { when } M \bar{m} \leq q_{\alpha} \\
& \text { where } q_{t} \text { is the } t \text {-quantile of a Poisson variable whose parameter is given by } M \hat{m}_{g}= \\
& 2 M \delta T \hat{\lambda}_{1} \hat{\lambda}_{2} \text {. }
\end{aligned}
$$

Up to our knowledge, no other operational method based on multiple shift coincidence count has been developed. In particular, the distribution free methods such 
as trial-shuffling methods developped by Pipa and collaborators, which avoid plug-in problems, are based on binned coincidence count (Pipa \& Grün, 2003; Pipa et al., 2003) and not on multiple shift coincidence count. Plug-in effects can also be avoided in another way, on binned coincidence count, by considering conditional distribution (Gütig et al., 2001).

\section{Simulation study on one window}

The simulation study is consequently restricted to the two previous sort of tests (GAUE and UE) to focus on this particular notion of delayed/multiple shift coincidence count, which is drastically different from binned coincidence count.

Simulated processes Several processes have been simulated. The Poisson processes have already been described in Section 2.2. They constitute a particular case of more general counting processes, called the Hawkes processes, which can be simulated by thinning algorithms (Daley \& Vere-Jones, 2003; Ogata, 1981; Reimer et al., 2012). After a brief apparition in (Chornoboy et al., 1988), they have recently been used again to model spike trains in (Krumin et al., 2010; Pernice et al., 2011, 2012). A bivariate Hawkes process $\left(N_{1}, N_{2}\right)$ is described by its respective conditional intensities with respect to the past, $\left(\lambda_{1}(),. \lambda_{2}().\right)$. Informally, the quantity $\lambda_{j}(t) d t$ gives the probability that a new point on $N_{j}$ appears in $[t, t+d t]$ given the past. We refer the reader to (Brown et al., 2002) for a more precise definition. General bivariate Hawkes processes are given for all time $t$ and all indexes $i \neq j$ in $\{1,2\}$ by

$$
\lambda_{j}(t)=\left(\nu_{j}+\int_{u<t} h_{j \rightarrow j}(t-u) N_{j}(d u)+\int_{u<t} h_{i \rightarrow j}(t-u) N_{i}(d u)\right)_{+} .
$$

The $\nu_{j}$ 's are real parameters called the spontaneous parameters. The functions $h_{j \rightarrow j}$, representing self-interaction, and $h_{i \rightarrow j}$, representing the interaction of neuron $i$ on neuron $j$, are functions with support in $\mathbb{R}_{+}$and are called the interaction functions. This equation means in particular that before the first occurrence of a spike, the $N_{j}$ 's behave like homogeneous Poisson processes with intensity $\nu_{j}$. The first occurrence of a spike (and the next ones) affects all the processes by increasing or decreasing the conditional intensities via the interaction functions.

For instance, if $h_{i \rightarrow j}$ takes large positive values in the neighborhood of a delay $x=5 \mathrm{~ms}$ and is null elsewhere, then $5 \mathrm{~ms}$ after after a spike in $N_{i}$, the probability to have a new spike in $N_{j}$ will significantly increase: the process $N_{i}$ excites the process $N_{j}$. On the contrary, if $h_{i \rightarrow j}$ is negative around $x$, then $5 \mathrm{~ms}$ after a spike in $N_{i}$, the probability to have a new spike in $N_{j}$ will significantly decrease: the process $N_{i}$ inhibits the process $N_{j}$. So Hawkes processes enable us to model lack of coincidences as well as 
profusion of coincidences depending on the sign of the interaction functions. The processes $\left(N_{1}, N_{2}\right)$ in this Hawkes model are independent if and only if $h_{1 \rightarrow 2}=h_{2 \rightarrow 1}=0$. Note also that the self-interaction functions $h_{j \rightarrow j}$, when very negative at short range, model refractory periods, making the Hawkes model more realistic than Poisson processes with respect to real data sets, even in the independence case. In particular when $h_{j \rightarrow j}=-\nu_{j} \times \mathbf{1}_{[0, x]}$, all the other interaction functions being null, the couple of simulated processes are independent Poisson processes with dead time $x$ (PPD), modeling strict refractory periods of length $x$ (Reimer et al., 2012). Finally Hawkes processes are not discretized at any resolution level as well as Poisson processes.

Another popular example is the injection model, which is discretized at the resolution $h$ and which is used in (Grün et al., 1999). Two independent Bernoulli processes $N_{B_{1}}$ and $N_{B_{2}}$ are generated with respective firing rates $\nu_{1}$ and $\nu_{2}$. Then a third Bernoulli process $N_{c}$ is generated with firing rate $\nu_{c}$. A fourth point process $N_{c}^{\prime}$ is generated from $N_{c}$ by moving independently each point of $N_{c}$ by a random uniform shift in $\{-x, \ldots, x\} \times h$, for a prescribed nonnegative integer $x$. Then the two spike trains are given by $N_{1}=N_{B_{1}} \cup N_{c}$ and $N_{2}=N_{B_{2}} \cup N_{c}^{\prime}$ (see (Grün et al., 1999) for more details). This injection model can in particular only model profusion of coincidences and not lack of coincidences.

Injection and Hawkes models are stationary, which means that their distribution does not change by translation in time (see (Daley \& Vere-Jones, 2003, p 178) for a more precise definition). This is also the case of homogeneous Poisson processes. One can also simulate inhomogeneous Poisson processes, which correspond to a conditional intensity $t \rightarrow \lambda(t)$ which is deterministic but not constant. These inhomogeneous Poisson processes are therefore non stationary in time (see (Daley \& Vere-Jones, 2003) for more details).

Figure 4 gives the average number of rejections over various numerical experiments, that have been led on those simulated processes.

Type I error First of all, let us inspect the level of the various tests in Figure 4.A and 4.B. We see that the three forms of GAUE (symmetric, upper and lower value) guarantee a level of roughly $5 \%$ and this even for a very small number of trials $(M=20)$ with a very small firing rate $(\lambda=3 \mathrm{~Hz})$ or with large $\delta(\delta=0.02 \mathrm{~s})$. In this sense, it clearly extends the validity of the UE method, which is known to be inadequate for firing rates less than $7 \mathrm{~Hz}$ (Roy et al., 2000) (see Experiments A and B). This level is also robust to changes in the model: non stationarity for inhomogeneous Poisson processes (Experiments C), refractory periods when using Hawkes processes (Experiments D and E). 

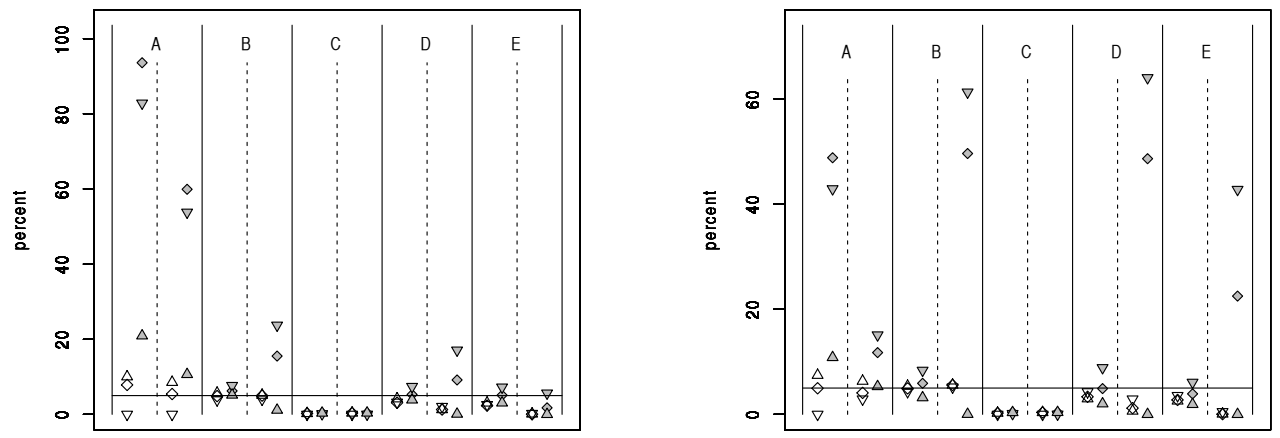

A

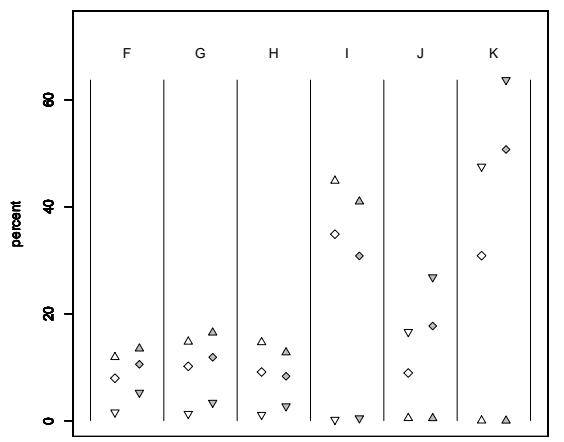

B

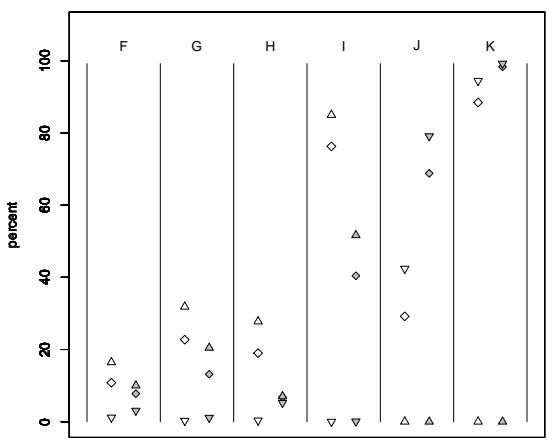

C

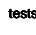

D

Figure 4: Average number of rejections of the independence hypothesis over $5000 \mathrm{nu}$ merical experiments over a window $W$ of length $T=0.1 \mathrm{~s}$. The diamonds, triangles up and down correspond respectively to symmetric tests, tests by upper value and tests by lower value, with $\alpha=0.05$. The white color corresponds to GAUE and the gray color to UE. In the 4 graphs, each column corresponds to a different kind of experiment, denoted by the letter on the top. In graphs $\mathbf{A}$ and $\mathbf{B}$, which correspond to Type I error, each column is subdivised into two smaller columns: in the left sub-column, the delayed coincidence count is computed with $\delta=0.005 \mathrm{~s}$, and in the right sub-column, with $\delta=0.02 \mathrm{~s}$. Graph A corresponds to a number of trials $M=20$ and graph $\mathbf{B}$ corresponds to $M=100$. On both graphs, the horizontal line corresponds to the level $\alpha=0.05$. Graphs $\mathbf{C}$ and $\mathbf{D}$ correspond to estimated power with $M=20$ and respectively $\delta=0.005 \mathrm{~s}$ and $\delta=0.02 \mathrm{~s}$. Experiments $\mathrm{A}$ and B correspond to independent homogeneous Poisson processes with respectively $\lambda_{1}=\lambda_{2}=3 \mathrm{~Hz}$ and $\lambda_{1}=\lambda_{2}=30 \mathrm{~Hz}$. Experiments $\mathrm{C}$ correspond to independent inhomogeneous Poisson processes with intensity $\lambda_{1}(t)=\lambda_{2}(t)=300 \times t+15$. Experiments $\mathrm{D}$ and $\mathrm{E}$ correspond to Hawkes processes with $\nu_{1}=\nu_{2}=30 \mathrm{~Hz}$ and with respectively $h_{1 \rightarrow 1}=h_{2 \rightarrow 2}=-10 \times \mathbf{1}_{[0,0.02]}$ and $h_{1 \rightarrow 1}=h_{2 \rightarrow 2}=-30 \times \mathbf{1}_{[0,0.02]}$, the other interaction functions being null. Experiments $\mathrm{F}$ and $\mathrm{G}$ correspond to injection models with resolution $h=10^{-4} \mathrm{~s}, \nu_{1}=\nu_{2}=30 \mathrm{~Hz}$, $x=200$ and respectively $\nu_{c}=5$ and $\nu_{c}=10$. Experiments $\mathrm{H}, \mathrm{I}, \mathrm{J}$ and $\mathrm{K}$ correspond to Hawkes processes with $\nu_{1}=\nu_{2}=30 \mathrm{~Hz}$ and only one non zero interaction function given by respectively $h_{2 \rightarrow 1}=10 \times \mathbf{1}_{[0,0.02]}, h_{2 \rightarrow 1}=30 \times \mathbf{1}_{[0,0.02]}, h_{2 \rightarrow 1}=-10 \times \mathbf{1}_{[0,0.02]}$ and $h_{2 \rightarrow 1}=-30 \times \mathbf{1}_{[0,0.02]}$. 
Power Several dependence situations have been tested in Figure 4.C and 4.D. We see that GAUE tests by upper value can adequately detect profusion of coincidences induced by injection models (see Experiments F and G) or Hawkes models (see Experiments $\mathrm{H}$ and I). GAUE tests by lower value can on the contrary detect lack of coincidences, simulated by inhibitory Hawkes processes (see Experiments $\mathbf{J}$ and E). Note moreover that symmetric GAUE tests can detect both situations.

As a partial conclusion, the Gaussian Approximation needs to be modified to take into account the plug-in effect. Once this modification has been done the Gaussian approximation leads to tests that are shown to be of asymptotic level $\alpha$. A simulation study has shown that those tests are robust to variations in the model (non stationarity, refractory periods), moreover symmetric GAUE tests are able to detect both profusion and lack of coincidences.

\subsection{Multiple tests and False Discovery Rate}

Classical UE analysis is performed on several windows, so that dependence regions can be detected through time. We want to produce the same kind of analysis with GAUE. However, since a test is by essence a random answer, it is not true that the control of one test at level $\alpha$ automatically induces a controlled of the number of false positive.

Indeed, let us consider a collection $\mathcal{W}$ of possibly overlapping windows $W$, with cardinality $K$ and, to illustrate the problem, let us assume that we observe two independent homogeneous Poisson processes. Now let us perform any of the previous GAUE tests at level $\alpha$ on each of the previous windows. Then by linearity of the expectation, one has that

$$
\mathbb{E}(\text { number of rejections })=K \mathbb{P}(\text { one test rejects }) \rightarrow_{M \rightarrow \infty} K \alpha \text {. }
$$

Moreover, if $L$ is the maximal number of disjoint windows in $\mathcal{W}$, then the probability that the $K$ tests accept the independence hypothesis is upper bounded by

$$
\mathbb{P}(\text { the } L \text { tests accept })=\mathbb{P}(\text { one test accepts })^{L} \rightarrow_{M \rightarrow \infty}(1-\alpha)^{L} \rightarrow_{L \rightarrow \infty} 0,
$$

by independence of the test statistics between disjoint windows. This means that for large $M$, this procedure is doomed to reject in average $K \alpha$ tests and the procedure will reject at least one test, when $L$ grows. Consequently, one cannot apply multiple tests procedure without correcting them for multiplicity. Ventura also underlined the problem of the multiplicity of the tests, and proposed a procedure which is not as general as the one described here (Ventura, 2010). 


\section{Multiple testing correction: a Benjamini and Hochberg approach}

Let us denote $\Delta_{W}$ the test considered on the window $W$.

One way to control multiple testing procedure based on the $\Delta_{W}$ 's, is to control the so called Family Wise Error Rate (FWER) (Hochberg \& Tamhame, 1987), which consists in controlling

$$
F W E R=\mathbb{P}\left(\exists W \in \mathcal{W}, \Delta_{W} \text { wrongly rejects }\right) .
$$

This can be easily done by Bonferroni bounds:

$$
\mathbb{P}\left(\exists W \in \mathcal{W}, \Delta_{W} \text { wrongly rejects }\right) \leq \sum_{W \in \mathcal{W}} \mathbb{P}\left(\Delta_{W} \text { wrongly rejects }\right) \rightarrow_{M \rightarrow \infty} K \alpha .
$$

So Bonferroni's method (Holm, 1979) consists in applying the $\Delta_{W}$ tests at level $\alpha / K$ instead of $\alpha$ to guarantee a FWER less than $\alpha$. However, the smaller the Type I error, the more difficult it is to make a rejection. Usually, the rejected tests are called detections (or discoveries). So when $K$ is large, Bonferroni's procedure potentially leads to no discovery/detection at all, even in cases where dependent structures exist.

Another notion, popularized by (Benjamini \& Hochberg, 1995) has consequently been introduced in the multiple testing areas leading to a large amount of publications in statistics, genomics, medicine etc in the past ten years (Benjamini, 2010). This is the False Discovery Rate (FDR). Actually, a false discovery (also named false detection or false positive) is not that bad if the ratio of the number of false discoveries divided by the total number of discoveries is small.

More formally, let us use the notation given in Table 1.

\begin{tabular}{c||c|c|c|} 
Number of $W$ such that & $\Delta_{W}$ accepts & $\Delta_{W}$ rejects & Total \\
\hline \hline Independence on $W$ & $\begin{array}{c}T_{n}=\text { " number of } \\
\text { true negatives" }\end{array}$ & $\begin{array}{c}F_{p}=\text { "number of } \\
\text { false positives" }\end{array}$ & $\begin{array}{c}K_{0}=\text { "number of windows } \\
\text { where independence is satisfied" }\end{array}$ \\
\hline Dependence on $W$ & $\begin{array}{c}F_{n}=\text { "number of } \\
\text { false negatives" }\end{array}$ & $\begin{array}{c}T_{p}=\text { "number of } \\
\text { true positives" }\end{array}$ & $\begin{array}{c}K_{1}=\text { "number of windows } \\
\text { where dependence exists" }\end{array}$ \\
\hline Total & $K-R$ & $R=$ "discoveries" & $K$ \\
\hline
\end{tabular}

Table 1: Repartition of the answers for the multiple testing procedure.

Then the False Discovery Rate is defined by

$$
F D R=\mathbb{E}\left(\frac{F_{p}}{R} \mathbf{1}_{R>0}\right) \text {. }
$$


Note that when both spike trains are independent for all windows, $K_{1}=0$, which leads to $T_{p}=0$ and $F_{p}=R$. Hence, the FDR in the full independent case is also a control of $\mathbb{P}\left(\exists W \in \mathcal{W}, \Delta_{W}\right.$ wrongly rejects $)$, i.e. the FWER. In all other cases, $F D R \leq F W E R$. This means that when there are some $W$ for which the independence assumption does not hold, controlling the FDR is less stringent, whereas the relative confidence that we can have in the discoveries is still good: if we make 100 discoveries with a FDR of $5 \%$, this means that on average only 5 of those discoveries will be potentially wrong.

The question now is: how to guarantee a small FDR? To do so, Benjamini and Hochberg (Benjamini \& Hochberg, 1995) proposed the following procedure: for each test $\Delta_{W}$, the corresponding $\mathrm{p}$-value $P_{W}$ is computed. They are next ordered such that:

$$
P_{W(1)}^{(1)} \leq \ldots \leq P_{W(\ell)}^{(\ell)} \leq \ldots \leq P_{W(K)}^{(K)}
$$

Let $q \in[0,1]$ be a fixed upper bound that we desire on the FDR and define:

$$
k=\max \left\{\ell \text { such that } P_{W(\ell)}^{(\ell)} \leq \ell q / K\right\} .
$$

Then the discoveries of this BH-method are given by the windows $W(1), \ldots, W(k)$ corresponding to the $k$ smallest $\mathrm{p}$-values.

The theoretical result of (Benjamini \& Hochberg, 1995) can be translated in our framework as follows: if the p-values are uniformly and independently distributed under the null hypothesis, then the procedure guarantees a FDR less than $q$.

Now let us finish to describe our method named MTGAUE, for Multiple Testing based on a Gaussian Approximation of the Unitary Events, which is based on symmetric tests to be able to detect both profusion and lack of coincidences.

\footnotetext{
Definition 4: MTGAUE

- For each $W$ in the collection $\mathcal{W}$ of possible overlapping windows, compute the p-value of the symmetric GAUE test (see Definition 2).

- Order those p-values according to (30) and find $k$ satisfying (31).

- Return as set of detections, the $k$ windows corresponding to the $k$ smallest $\mathrm{p}$ values.
}

Note that in our case, the assumptions required in the approach of BenjaminiHochberg are not satisfied. Indeed, the tests $\Delta_{G A U E}$ are only asymptotically of Type I error $\alpha$, which is equivalent to the fact that asymptotically and not for fixed $M$, the p-values are uniformly distributed. Therefore, there is a gap between theory and what we have in practice. However, as we illustrate hereafter in simulations, this difference does not seem to significantly impact the FDR.

Moreover, to have independent $\mathrm{p}$-values we should have considered disjoint windows $W$. However, it is possible that we miss some detections because the disjoint windows 
would not be correctly centred. Therefore, it is preferable to consider sliding windows that overlap. In theory, few results exist in this context - see for instance (Benjamini \& Yekutieli, 2001). In practice, we will see in the next section that this lack of independence does not impact the FDR as well.

\section{Simulation study}

On Figure 5, we see an example of detection by both methods: MTGAUE and UE. Note that UE is here performed without corrections due to the multiplicity of the tests as in (Grün et al., 1999) and therefore its parameter is $\alpha$, which should reflect the level of each individual test. MTGAUE clearly detects relevant regions and very few false positives and this even for large delays $\delta$, with a clear continuity in $\delta$ : once a region has been detected for a small $\delta$, it remains generally detected for a larger $\delta$, until the number of imposed coincidences is diluted because $\delta$ is much too large. Therefore there is usually an interval of possible $\delta$ around the actual maximal true interaction (here $0.02 \mathrm{~s}$, whatever the model, injection or Hawkes) where the same window is detected. Note also that the positive interactions in Hawkes model or injection model (i.e. profusion of coincidences) are clearly detected and that the detected regions correspond to positive values for the difference $\bar{m}-\hat{m}_{0}$. Reciprocally negative interactions in Hawkes model (i.e. lack of coincidences) are also detected and correspond to negative values of $\bar{m}-$ $\hat{m}_{0}$, since they appear on Figure 5.C but not on 5.D.

Figure 6 gives the average proportion of false positive (FDR) and false negative. Clearly MTGAUE ensures a FDR less than $5 \%$ as expected by the choice of the parameter $q=0.05$ and this, in all simulations. Moreover the proportion of false negative is relatively small (less than 30\%) and clearly decreases when $M$ the number of trials increases. Note also than even if the trials are not i.i.d. (Experiment D), the method still guarantees a controlled FDR and a reasonable amount of false negative.

\section{Real data study}

MTGAUE being validated on simulated data, the method is now applied on real data, that have already been partially published. This study is an illustration which shows that MTGAUE is able to detect phenomenons in adequation with the time of the experiment. Furthermore some novel aspects are revealed thanks to this method, completing the existing results on those data. 


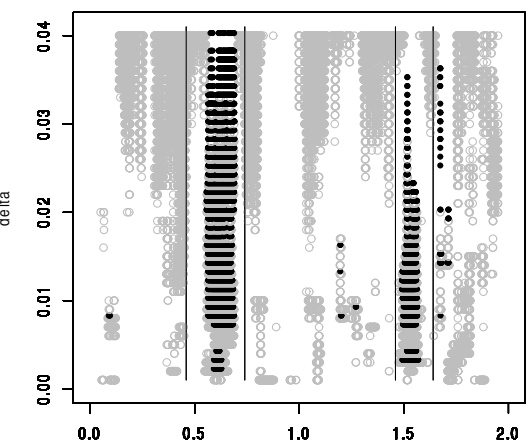

A

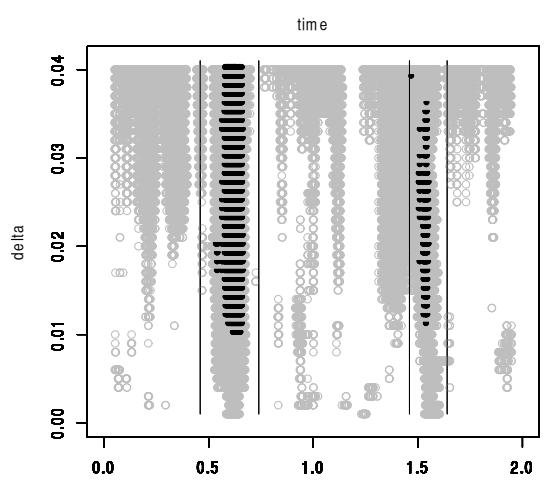

B

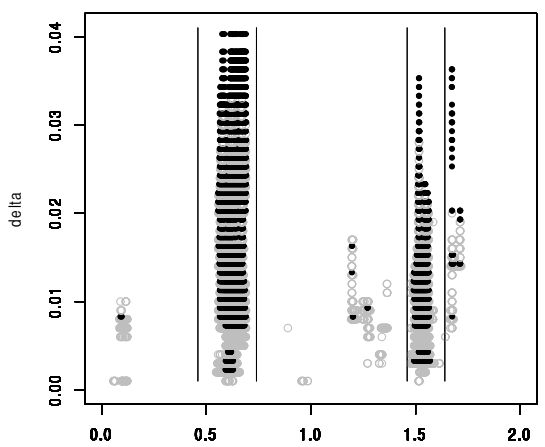

C

time

D
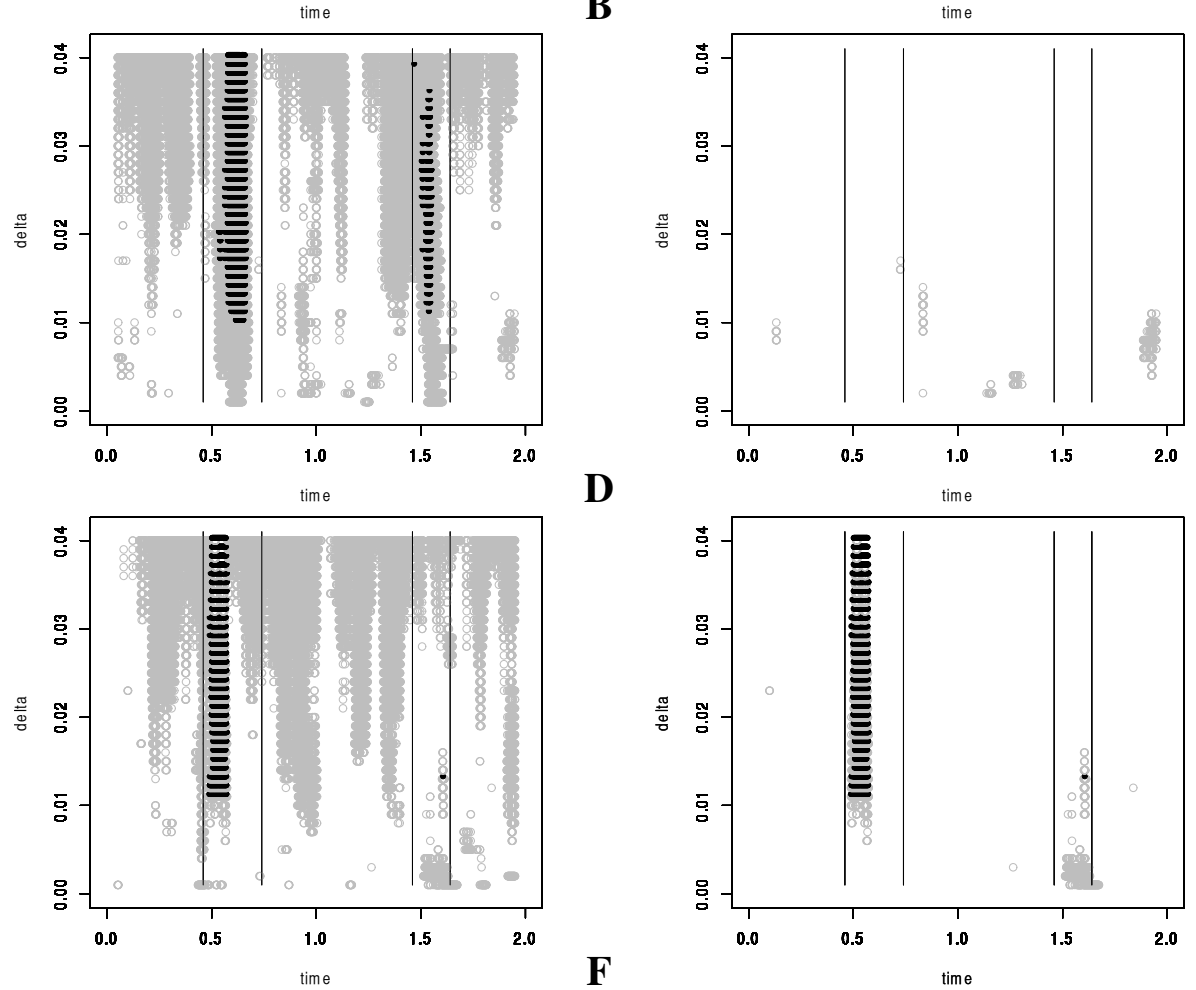

$\mathbf{D}$

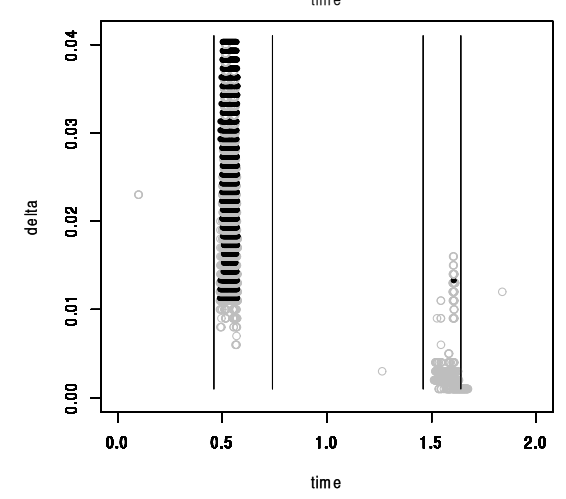

Figure 5: Detections of MTGAUE with $q=0.05$ (black dots) and of the symmetric UE method with $\alpha=0.05$ (gray circles), on one run, in $\mathbf{A}, \mathbf{C}, \mathbf{E}$, with $M=20$. In $\mathbf{B}, \mathbf{D}, \mathbf{F}$, same respective experiments, but only the detections for which $\bar{m}>\hat{m}_{0}$ for MTGAUE, or detections for which $\bar{m}>m_{g}$ for UE, are represented. 1900 single tests have been performed on 1900 overlapping (sliding) windows of length $0.1 \mathrm{~s}$ shifted by $0.001 \mathrm{~s}$. The corresponding detection is marked by a dot/circle at the center of the window. Each line corresponds to a different delay: 40 different delays from $0.001 \mathrm{~s}$ to $0.04 \mathrm{~s}$ are considered. Each time, two homogeneous independent Poisson processes are simulated on $[0,0.5] \cup[0.7,1.5] \cup[1.6,2]$ with firing rates $\lambda_{1}=\lambda_{2}=30 \mathrm{~Hz}$. On $[0.5,0.7]$ and $[1.5,1.6]$ two dependent processes are simulated. The black vertical lines delimit the regions where the tests should detect a dependence, that is each time the window intersects a dependence region. In $\mathbf{A}, \mathbf{B}$, the dependent processes are Hawkes processes with $\nu_{1}=\nu_{2}=30 \mathrm{~Hz}$ and $h_{1 \rightarrow 2}=30 \times \mathbf{1}_{[0,0.02]}$, the other interaction functions being null. In $\mathbf{C}$, $\mathbf{D}$, the same with $h_{1 \rightarrow 2}=-30 \times \mathbf{1}_{[0,0.02]}$. In $\mathbf{E}, \mathbf{F}$, the dependent processes are given by an injection model with resolution $h=10^{-4} \mathrm{~s}$ with $\nu_{1}=\nu_{2}=30 \mathrm{~Hz}$, $x=200$ and $\nu_{c}=10$. 


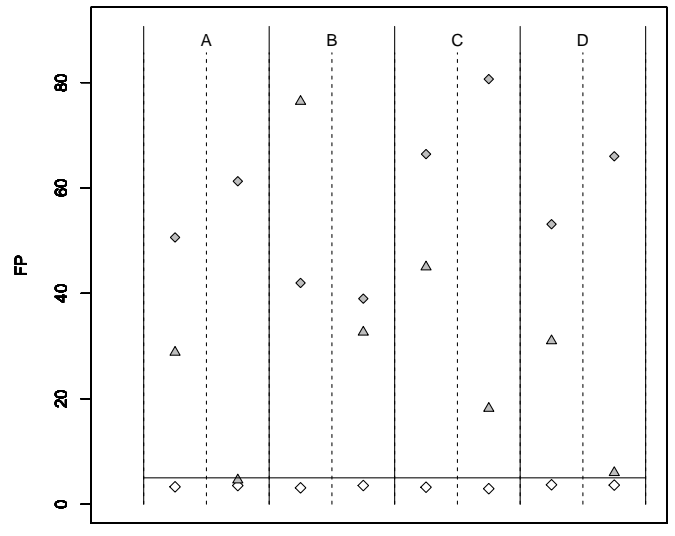

A

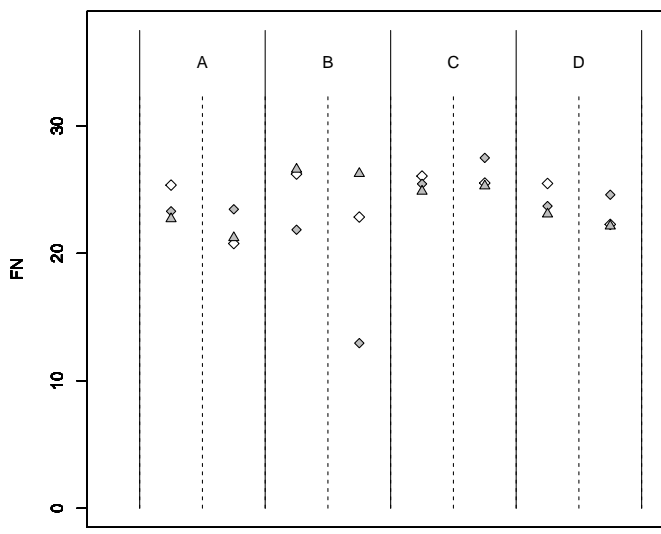

C

tests

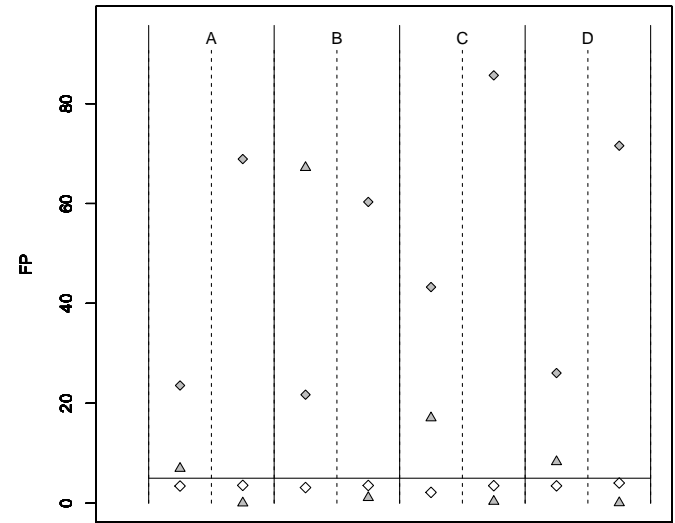

B

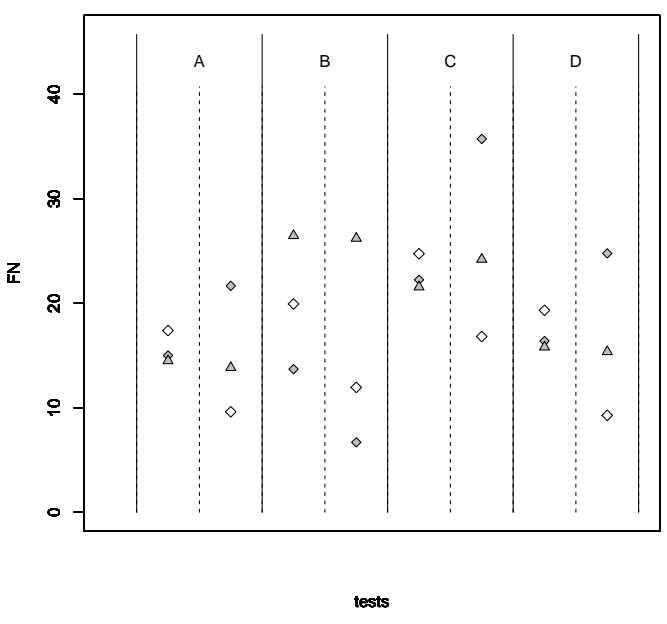

Figure 6: Average over 5000 runs of the ratio $F_{p} / R$ (see Table 1) giving the false discovery rate in $\mathbf{A}$ and $\mathbf{B}$, and of the ratio $F_{n} /(K-R)$ giving the proportion of false negative in $\mathbf{C}$ and $\mathbf{D}$. The white diamond corresponds to MTGAUE with $q=0.05$, the gray diamond to the symmetric UE method with $\alpha=0.05$, the gray triangle to the detections of the symmetric UE method for which $\bar{m}>m_{g}$. Graphs $\mathbf{A}$ and $\mathbf{C}$ correspond to $M=20$, whereas graphs $\mathbf{B}$ and $\mathbf{D}$ correspond to $M=100$. On graphs $\mathbf{A}$ and $\mathbf{B}$, an horizontal line at the level 0.05 is plotted. Each column corresponds to an experiment, the left sub-column corresponding to $\delta=0.005 \mathrm{~s}$ and the right subcolumn to $\delta=0.02 \mathrm{~s}$. Experiments A, B and C correspond respectively to the same set-up as Figures 5.A, 5.C, 5.E. Experiments D correspond to non i.i.d. trials. More precisely, 50\% of the trials are simulated according to Experiments A, 25\% of the trials are pure independent Poisson processes with intensity $\lambda_{1}=\lambda_{2}=30 \mathrm{~Hz}, 25 \%$ trials are similar to Experiments A except that the dependence regions are now $[0.55,0.75]$ and $[1.45,1.6]$. The references for false positive/false negative in Experiments D are given by Experiments A.

\subsection{Description of the data}

Behavioral procedure The data used in this theoretical article to test the detection ability of the MTGAUE method were already partially published in previous experi- 
mental studies (Riehle et al., 2000; Grammont \& Riehle, 2003; Riehle et al., 2006). These data were collected on a 5-year-old male Rhesus monkey who was trained to perform a delayed multidirectional pointing task. The animal sat in a primate chair in front of a vertical panel on which seven touch-sensitive light-emitting diodes were mounted, one in the center and six placed equidistantly (60 degrees apart) on a circle around it. The monkey had to initiate a trial by touching and then holding with the left hand the central target. After a delay of 500ms, the preparatory signal (PS) was presented by illuminating one of the six peripheral targets in green. After a delay of either 600 or $1200 \mathrm{~ms}$, selected at random with various probability, it turned red, serving as the response signal and pointing target. During the first part of the delay, the probability for the response signal to occur at $500+600 \mathrm{~ms}=1.1 \mathrm{~s}$ was either $0.3,0.5$ or 0.7 , depending on the experimental condition. Once this moment passed without signal occurrence, the conditional probability for the signal to occur at $500+600+600 \mathrm{~ms}=1.7 \mathrm{~s}$ changed to 1. The monkey was rewarded by a drop of juice after each correct trial. Reaction time (RT) was defined as the release of the central target. Movement time (MT) was defined as the touching of the correct peripheral target.

Recording technique Signals recorded from up to seven microelectrodes (quartz insulated platinumtungsten electrodes, impedance: $25 \mathrm{M} \Omega$ at $1000 \mathrm{~Hz}$ ) were amplified and band-pass filtered from $300 \mathrm{~Hz}$ to $10 \mathrm{kHz}$. Using a window discriminator, spikes from only one single neuron per electrode were then isolated. Neuronal data along with behavioral events (occurrences of signals and performance of the animal) were stored on a $\mathrm{PC}$ for off-line analysis with a time resolution of $10 \mathrm{kHz}$.

In the following study, only trials where the response signal occurs at $1.7 \mathrm{~s}$ are considered.

\subsection{Symmetric detections}

MTGAUE (see Definition 4) has been applied on the activity of two pairs of neurons, recorded during the experiment described in Section 4.1, for various choices for the FDR control parameter $q$. The classical multiple shift symmetric UE method with parameter $\alpha=0.05$ has also been applied. The results are displayed in Figure 7.

The black circles correspond to MTGAUE detections with $q=0.01$ and consequently at the most $1 \%$ of those detections are false positive in average. Those are the surest detections. The classical detections with $q=0.05$ correspond to the black and magenta circles. At the opposite, yellow circles correspond to untrusted detections since they are detected only for a FDR control parameter $q$ larger than $80 \%$. In this re- 
Values of the MTGAUE parameter $q$ over all considered windows

A
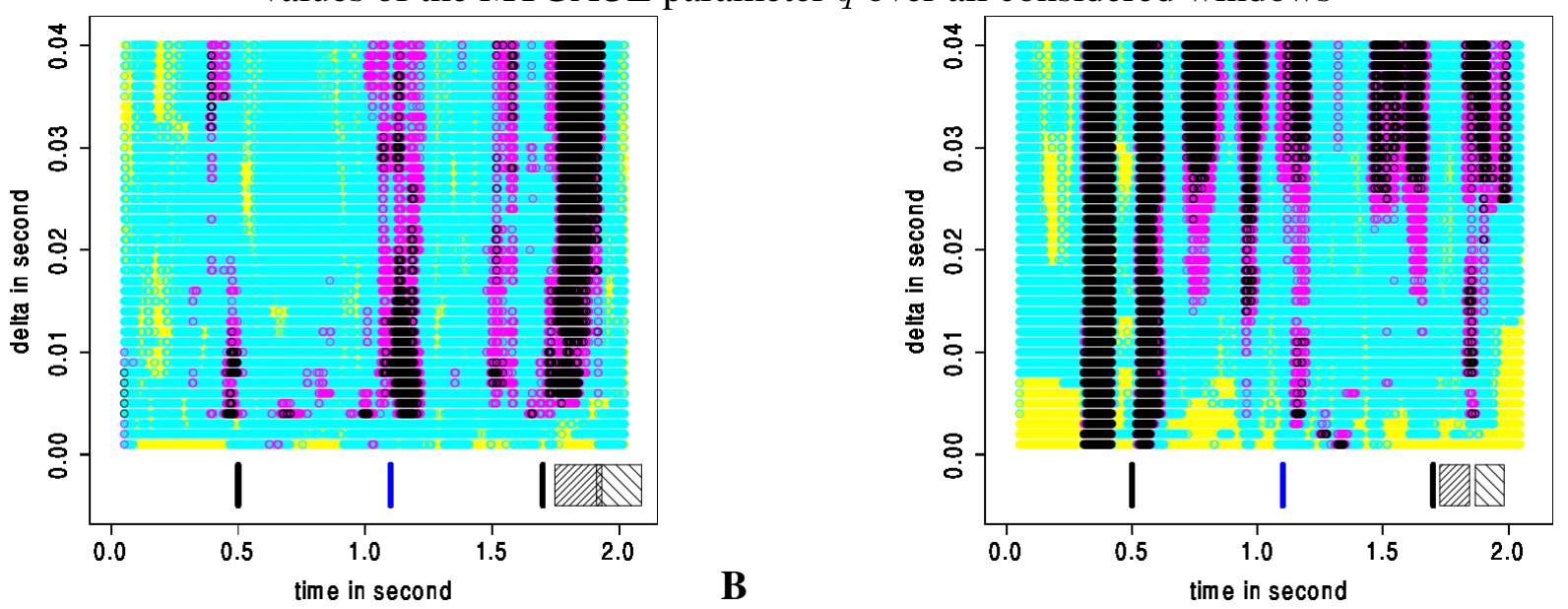

Values of the MTGAUE parameter $q$ for windows detected by the symmetric UE procedure with $\alpha=0.05$
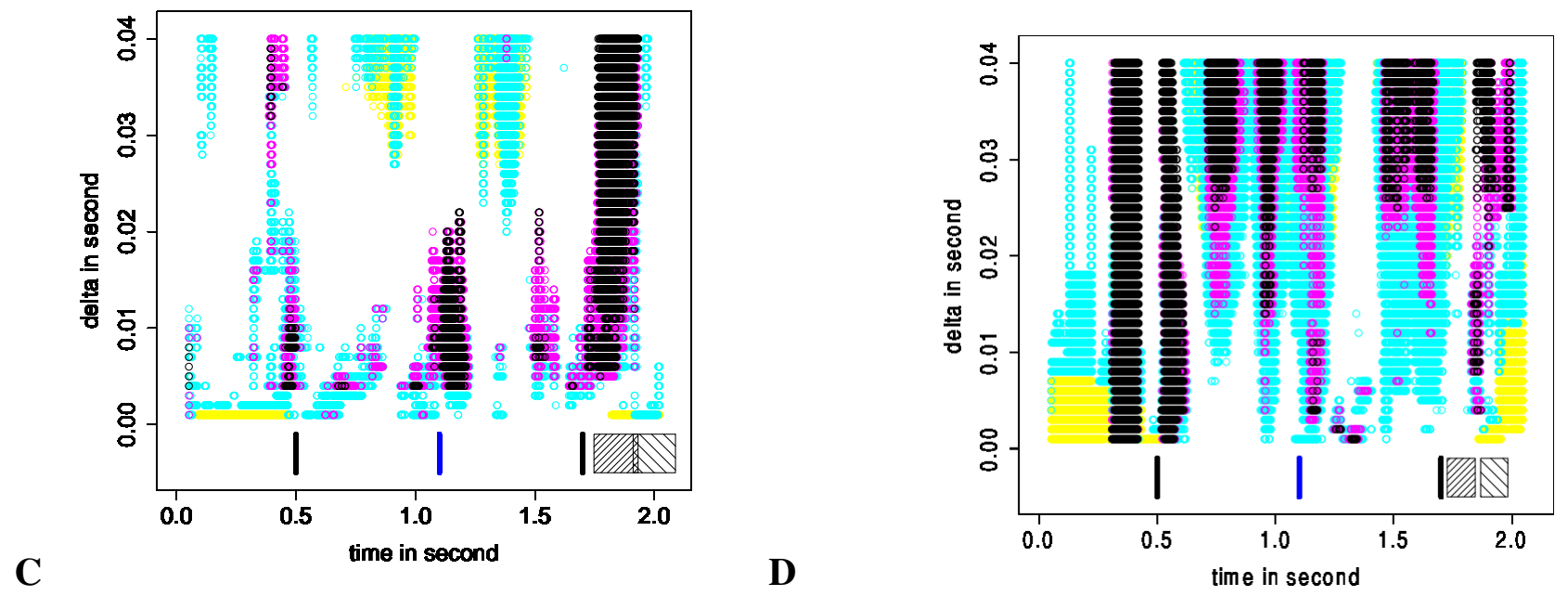

Figure 7: Representation of the detections over sliding windows of length $0.1 \mathrm{~s}$ shifted by $0.001 \mathrm{~s}$. Each window is associated to a color depending on the value of the parameter $q$ in the MTGAUE method. If it is detected for $q=0.01$, then a black circle is plotted at the center of the corresponding window. If it is not detected for $q=0.01$, but it is detected for $q=0.05$, then the color is magenta. If it is detected for $q=0.8$ but not for $q=0.01$ or $q=0.05$, the color is cyan. Yellow corresponds to none of the previous detections (meaning that the detection is for a parameter $q>0.8$ ). Each line corresponds to a different value of $\delta$ from 0.001 to $0.04 \mathrm{~s}$. The first black vertical bar corresponds to the preparatory signal (PS), the blue vertical bar to the expected signal (ES), the second black vertical bar to the response signal (RS). The first hatched box corresponds to the interval [mean reaction time (RT) minus its standard deviation, mean reaction time (RT) plus its standard deviation], the second hatched box corresponds to the same thing but for the movement time (MT). A and $\mathbf{C}$ correspond to the pair of neurons 13 in the experiment. B and D correspond to the pair of neurons 40 in the experiment. Graphs A and B display all the windows. Graphs $\mathbf{C}$ and $\mathbf{D}$ display the windows detected by the symmetric UE procedure with $\alpha=0.05$. 
spect, on 7.A and 7.B, several periods are detected by MTGAUE which correlate with the occurrence of specific events of the behavorial protocol. Interestingly, there is no real gain by looking at the detections for $q=0.05$. Most of them are already detected for $q=0.01$ for larger $\delta$ and therefore, these detections are really significant. On 7.C and 7.D, we see that the symmetric UE method with $\alpha=0.05$ detects several intervals corresponding to untrusted (yellow) detections by the MTGAUE method.

\subsection{Is the count significantly too low or too large?}

For single tests, the detection of $\Delta^{s y m}$ at level $\alpha=0.05$ is just the detection of both tests $\Delta^{+}$and $\Delta^{-}$at level $\alpha=0.025$ and this for both UE and GAUE methods (see Definitions 2 and 3). When dealing with a collection of tests, this result is still valid for the UE method which does not correct for multiplicity. However, it is not valid anymore for MTGAUE (see Definition 4) since this method is based on the rank of all the p-values of all the symmetric tests, somehow intertwining detections by lower and upper values. Since the interest lies in both distinct detections (upper and lower values) on the experimental data, it is meaningless to independently perform tests by upper values and then tests by lower values. The correct way to use the method is consequently to perform MTGAUE with the symmetric tests, as defined in Definition 4. Once a window is detected, one can then ask the question whether this detection was due to a low count or large count by looking at the sign of $\bar{m}-\hat{m}_{0}$, which is coherent with Figure 5.

Figure 8 shows the detections according to their signs. The UE detections by upper values are much more precise than the symmetric UE detections. The surest detections (in black) of MTGAUE in 7.C and 7.D that are not found in 8.A and in 8.B correspond to detections where the observed coincidence count is significantly too low, which can be detected by the symmetric MTGAUE method, but not by the UE method by upper values. In order to view the results directly on the data, the rasters associated to the data sets used in Figure 7 for $\delta=0.02 \mathrm{~s}$ are represented in Figures 8.C and 8.D, with in red the periods with profusion of coincidences and in blue the periods with lack of coincidences.

\subsection{Aggregation of the results obtained from several recording ses- sions}

We run the MTGAUE method over three different data sets, corresponding to the various probabilities of occurrence of the response signal at 1.1s. Once again, only trials where the response signal occurs at $1.7 \mathrm{~s}$ are selected for analysis. The main point here 


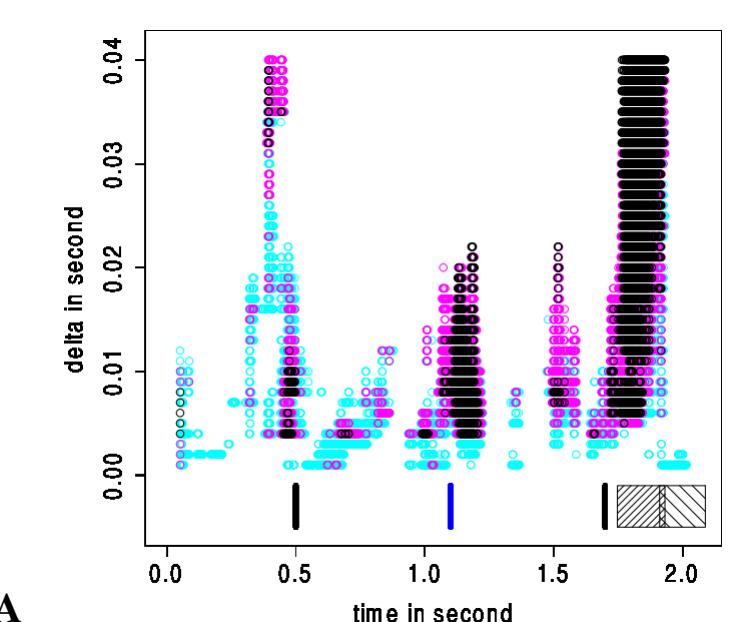

A

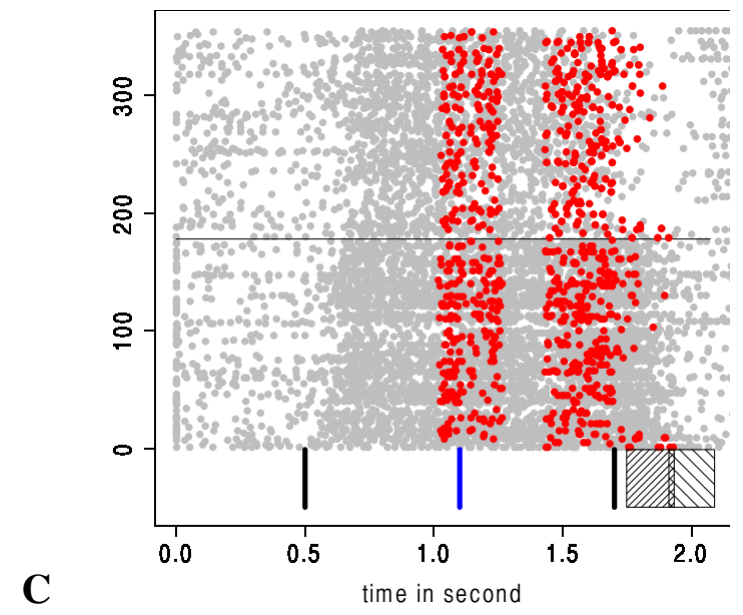

B
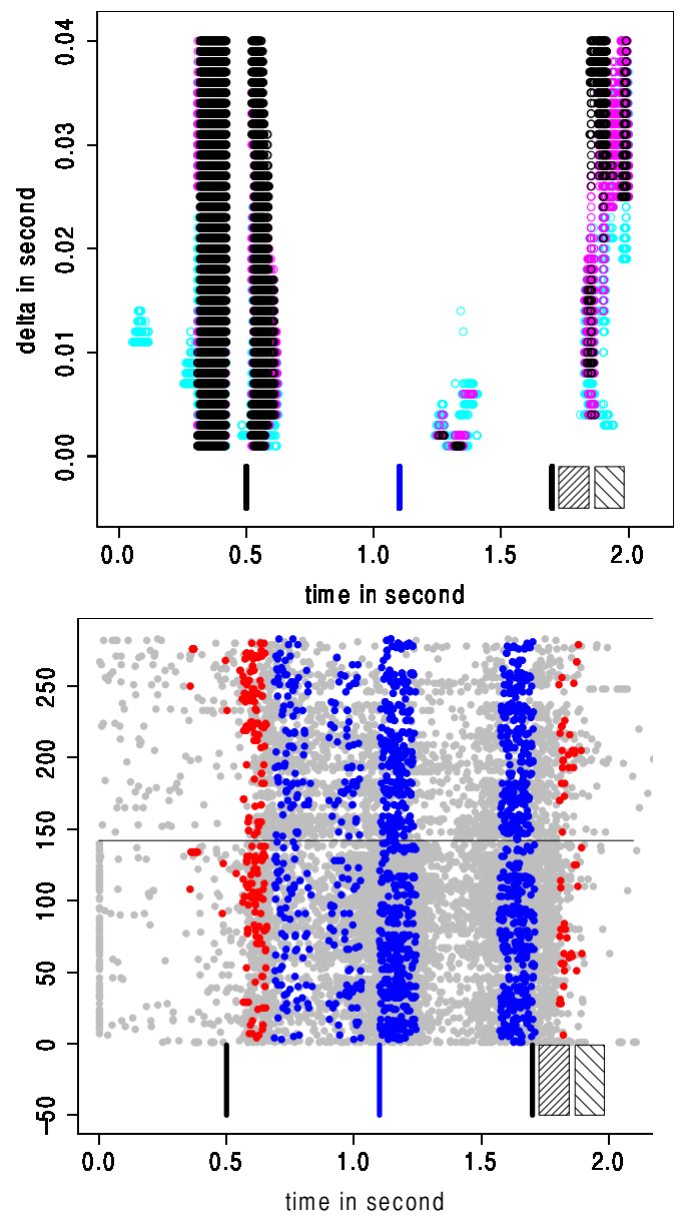

Figure 8: Detections according to the sign of the test statistics. In graphs $\mathbf{A}$ and $\mathbf{B}$, representation of the detections according to the symmetric UE method for which $\bar{m}>$ $\hat{m}_{g}$ with $\alpha=0.05$, the color being given by the value of the MTGAUE parameter $q$, with the same convention as in Figure 7. A corresponds to the couple of neurons 13 in the experiment. B corresponds to the couple of neurons 40 in our experiment. Graphs $\mathbf{C}$ and $\mathbf{D}$ are respectively the raster plot of couple 13 and couple 40 with in red the coincidences corresponding to a significantly too large coincidence count and in blue the coincidences corresponding to a significantly too low coincidence count, the detection being made by the symmetric MTGAUE with $q=0.05$ and $\delta=0.02 \mathrm{~s}$. Bars and boxes on the time axis as in Figure 7. 
A

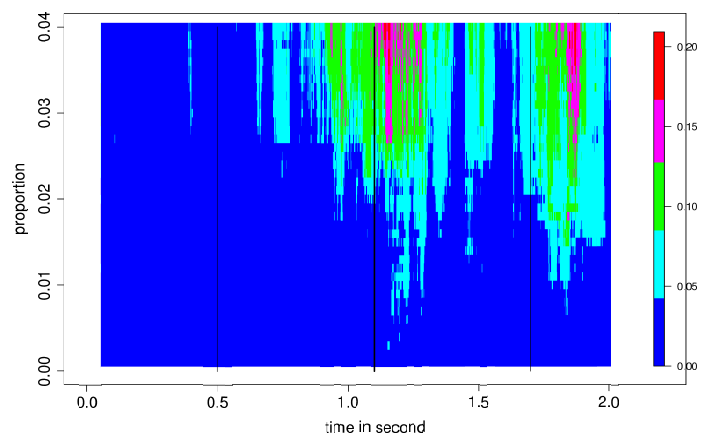

C

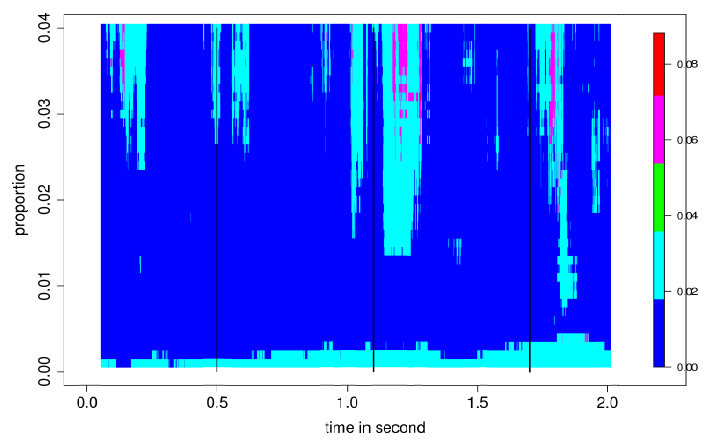

$\mathbf{E}$

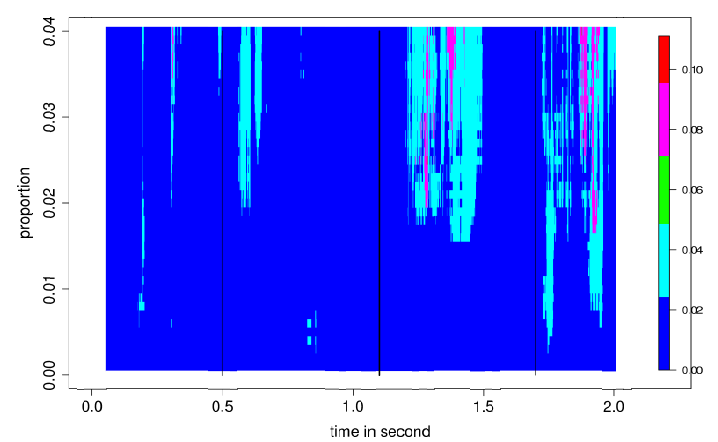

B

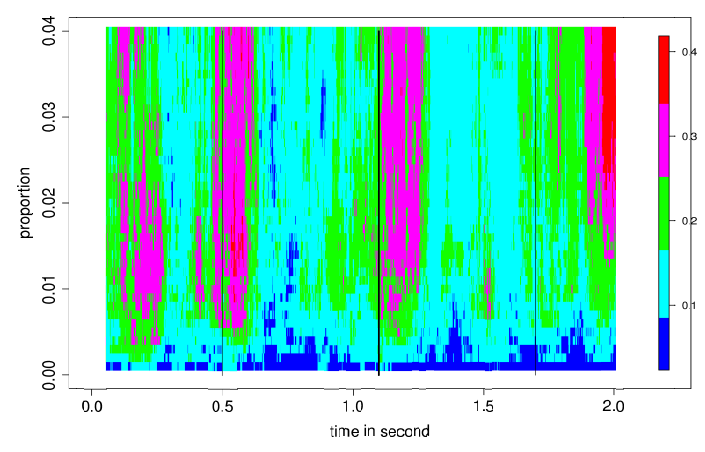

D

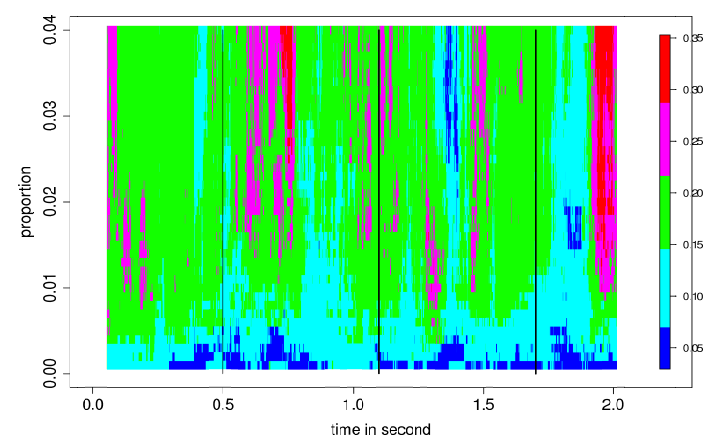

$\mathbf{F}$

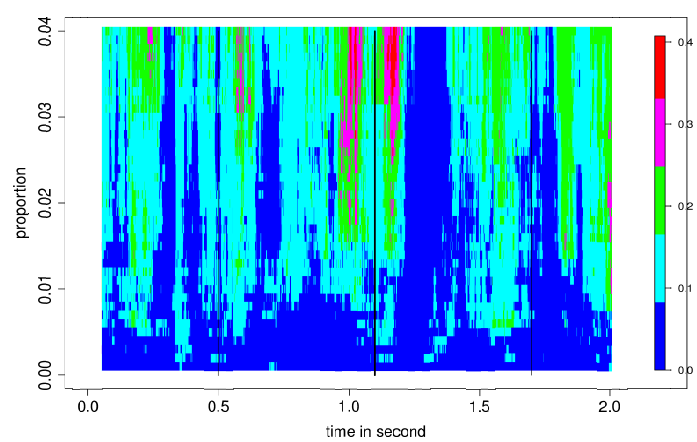

Figure 9: Proportion of pairs of neurons that have been detected by the MTGAUE method (sliding windows of length $0.1 \mathrm{~s}$ shifted by $0.001 \mathrm{~s}, q=0.05$ ) through time. Each line represents a different parameter $\delta \in\{1, \ldots, 40\} \times 10^{-3}$ s. A, $\mathbf{C}$ and $\mathbf{E}$ correspond to the proportion of pairs of neurons that have been detected by MTGAUE and for which the coincidence count is significantly too large whereas $\mathbf{B}, \mathbf{D}$ and $\mathbf{F}$ correspond to the proportion of pairs of neurons that have been detected by MTGAUE and for which the coincidence count is significantly too low. (PS) was presented at $0.5 \mathrm{~s}$ (first vertical black line), (RS) at 1.7s (third vertical black line). Even if those trials are not used in the present average, a response signal at $1.1 \mathrm{~s}((\mathrm{ES})=$ second vertical black line $)$ was also presented in $30 \%$ of the cases for A and B (Data30 - 43 pairs of neurons), in $50 \%$ of the cases for $\mathbf{C}$ and $\mathbf{D}$ (Data50 - 34 pairs of neurons) and in $70 \%$ of the cases in $\mathbf{E}$ and F (Data70 - 27 pairs of neurons). 
is to see the influence of the probabilities of occurrence of (RS) at 1.1s on the synchronized activity. Note that, despite the fact that this pool of data has already been published (Grammont \& Riehle, 2003), there has been no systematic analysis in terms of the impact of these various probabilities. There has been no systematic analysis of proportion of lack of coincidences either.

Because of the very small number of trials, for which the response signal occurs at $1.7 \mathrm{~s}$ (in particular in Data70), in a given movement direction, we decided to pool all directions together before analysis, as already done in the previous study but in a different way (Grammont \& Riehle, 2003). The trials are consequently not i.i.d. but Figure 6 shows that the MTGAUE detections can be trusted anyway. Moreover, we did not discard pairs of neurons whose firing rate is smaller than $7 \mathrm{~Hz}$ (Roy et al., 2000; Riehle et al., 2000; Grammont \& Riehle, 2003) because even in this case the MTGAUE detections can be trusted (see Figure 4). MTGAUE has been performed on each pair of neurons, leading to the detection of windows where this pair is significantly synchronized or anti-synchronized. Then, an aggregation of the detections over all pairs of each data set was performed. The proportion of these significantly synchronized/antisynchronized pairs are displayed in Figure 9. As already observed in (Grammont \& Riehle, 2003) and as a confirmation of our approach, the maximal proportions of significantly synchronized pairs of neurons occur in correlation with the occurrence of the different behavioral events of the task (preparatory, expected and response signals). There is a huge proportion of detections (up to $40 \%$ of the pairs) that are due to a coincidence count significantly too low, whereas at most, $10 \%$ of the pairs have a coincidence count significantly too large. In other terms, at a given moment of the task a maximum of about $10 \%$ of the pairs can be significantly synchronized and a maximum of $40 \%$ of them can be anti-synchronized. The fact that a bigger proportion of pairs can be anti-synchronized at a given moment of the task remains to be explained in neurophysiological terms and is beyond the scope of this article. Actually and as far as we know, although anti-synchronization can technically be studied more or less rigorously by most analysis method in this domain, none systematic study has been performed on this phenomenon from a more neurophysiological point of view. We know few examples where such anti-synchronizations are mentioned: in (Grammont \& Riehle, 1999), with the classical UE method based on binned coincidence count (Grün, 1996), and in (Riehle et al., 2000) with the UE method based on multiple shift coincidence count (Grün et al., 1999).

- Significantly too large coincidence count.

Comparing Figures $9 \mathbf{A}, \mathbf{C}$ and $\mathbf{E}$, it appears that the proportion of pairs that have a significantly too large coincidence count is much more important on Data30, 
showing two maxima, just after the expected signal and just after the response signal. When the probability of the expected signal increases (Data50 and Data70), those maxima still appear but in a lower proportion. Therefore it appears with this new analysis that the proportion of synchronized pairs tends to diminish when the probability of (RS) at 1.1s increases. Moreover, when the response signal at $1.1 \mathrm{~s}$ is much more likely than the response signal at 1.7s (in Data70), those maxima are shifted on the right as if the animal continued to expect the signal a longer time.

- Significantly too low coincidence count.

Comparing Figures $9 \mathbf{B}, \mathbf{D}$ and $\mathbf{F}$, it appears that all the three data sets have roughly the same maximal proportion of pairs that have a significantly too low coincidence count. The main difference comes from the localization and the fuzziness of the proportion of pairs. The maxima are large and strong in Data30 in particular around the preparatory and expected signals. In Data70, the only localizations reaching such high scores are just before and after the expected signal. In Data50, the signal is fuzzier and it is harder to give a rigorous interpretation.

\section{Discussion}

We presented a generalization, namely the delayed coincidence count, of the notion of multiple shift coincidence count (Grün et al., 1999) to point processes that are not discretized at a given resolution. This multiple shift notion clearly outperforms classical binned coincidence count, since there is no information loss due to binning effects as already pointed out in (Grün et al., 1999). However, this notion is not as popular as the binned coincidence count mainly because its statistical properties were much more opaque, until the present work. Indeed, the present generalization allows us to perform complete computations when Poisson processes are involved. It consequently also reveals some basic properties of the multiple shift coincidence count for Bernoulli processes, this model being very well approximated by Poisson processes as proved in Section 2.3.

The first novel property is that the multiple shift coincidence count, as well as the delayed coincidence count, is not Poisson distributed, but more dispersed, as shown by the Fano factor in (12), when the underlying processes are Poisson or Bernoulli. This gap tends to increase with the firing rate and the delay $\delta$, making the Poisson approximation performed in (Grün et al., 1999) not adequate for large $\delta(\delta=0.02 \mathrm{~s}$ ), as shown on Figure 2. Moreover, there is an edge effect that has been originally discarded and that needs to be taken into account (see again Figure 2 and Theorem 1), once again 
for large $\delta$. For small $\delta$, the Poisson approximation done in (Grün et al., 1999) is just less dispersed (see Figure 2).

Those properties would be useless, if they could not be turned into a real statistical test. To do so, we investigated the plug-in step, showing that a modification of the variance need to be taken into account (see Theorem 2). Our GAUE (for Gaussian Approximation of the Unitary Events) tests (see also Definition 2) are therefore proved to be of asymptotical level $\alpha$, when Poisson processes are considered as models of the spike trains. They are also proved to be robust on simulations where small firing rates, non stationarity or refractory periods have been imposed and this even for the test by lower value, which is able to detect lack of coincidences (see Figure 4). We also see that for small $\delta(\delta=0.005 \mathrm{~s})$ and large enough firing rate $\lambda(\lambda=30 \mathrm{~Hz})$, the three UE tests of (Grün et al., 1999) (see also Definition 3) have Type I error $\alpha=5 \%$. This is in the range of parameters where the original UE method with the multiple shift coincidence count, based on a Poissonian approximation, can be used.

For very small firing rates, the Type I errors of all UE tests are huge, fact which is consistent with (Roy et al., 2000) (see Figure 4, Experiments A). For large $\delta$ and large firing rates, the UE test by upper values becomes too conservative (see Figure 4, Experiments B). On the contrary, both UE tests (symmetric or by lower values) have very high Type I error (more than 20\%), and this can even increase with the number of trials $M$. This is coherent with Figure 3, where we clearly see that for large $\delta$ the distribution is not correctly centred, making the UE test by upper values more conservative but the other UE tests (symmetric and by lower values) untrustworthy. In this sense, even if UE tests (symmetric or by lower values) have larger power than GAUE tests for detecting lack of coincidences (see Figure 4, Experiments $\mathrm{J}$ and $\mathrm{K}$ ), they cannot be preferred to the present MTGAUE method since there is no control of their Type I error. Finally note that the UE method has level $\alpha=0.05$ when non stationary processes or refractory periods with small $\delta$ are tested (Experiments C, D and E). But both UE tests (symmetric and by lower values) with large $\delta$ have a Type I error which clearly becomes huge when $M$, the number of trials grows and when refractory periods are present (Experiments D and $\mathrm{E})$.

Two other methods in the literature do not need plug-in steps. On the one hand, conditional probability computations can be performed on binned coincidence count (Gütig et al., 2001), but it will be, in our opinion very difficult to transfer those computations to the delayed coincidence count, since the use of independent Bernoulli variables is fundamental. On the other hand, trial-shuffling methods (Pipa et al., 2003; Pipa \& Grün, 2003) have also been used for binned coincidence count. This kind of resampling methods could be adapted to the delayed coincidence count. However, the combinatorics of the delayed coincidence count is much more complex than the binned coincidence 
count. Hence, despite the use of trial-shuffling, which is the quickest way to generate surrogate data (Louis et al., 2010), the computational burden will be very high. Nevertheless, the use of surrogate data, to bypass dependency in the underlying model for the delayed coincidence count, is fundamental. Therefore, the adaptation of trial-shuffling to delayed coincidence count via adequate programming is one of the questions that deserves in our opinion a full and complete study in a future work.

The final step of our procedure is the use of Benjamini and Hochberg's method to control the False Discovery Rate (FDR) by the choice of a parameter $q$ (Benjamini \& Hochberg, 1995). Our method, MTGAUE, is proved on various simulations to guarantee the correct FDR (see Figure 6). On the contrary, the classical UE method is not corrected for multiplicity. Simulations show that this choice leads to a huge FDR, sometimes as high as $80 \%$. Only for large delays $\delta$ and only for the test by upper values, is the UE method trustworthy (in particular for values of the number of trials as large as 100). This is coherent with the visualisation of both methods on just one run (see Figure 5). Clearly for large $\delta$ the number of false positives by the symmetric UE is huge whereas the detections by upper values are globally adequate, with a tendency to be less accurate for small $\delta$. This phenomenon can be explained as follows. For large $\delta$ and reasonable firing rates, the UE test by upper values is too conservative (see Experiments B of Figure 4) because the edge effects have not been taken into account. Therefore the fact that the method is not corrected for the multiplicity of tests somehow compensates for its conservativeness leading in this precise situation to a reasonable method. But this happens only for this range of parameters and only for the detection of profusion of coincidences. In all other situations, there is no guarantee for the multiple shift UE method of (Grün et al., 1999).

To summarize those simulations, MTGAUE, which relies on a theoretical approximation of the distribution of the coincidence count by a Gaussian distribution, seems to be robust with respect to small firing rates, non stationarity in time, refractory periods and non ergodicity across trials. It guarantees a control of the FDR via the choice of the parameter $q$. This method is also able to detect both profusion and lack of coincidences. It therefore clearly extends the range of situations where the original multiple shift UE method applies, which is trustworthy only for delays of the order 0.02s (see Figure 6), reasonable firing rates $(\lambda>7 \mathrm{~Hz}$, see (Roy et al., 2000)) and detections when the coincidence count is significantly too large.

Therefore, when MTGAUE is applied on real data (already partly published in (Grammont \& Riehle, 2003)), it can in particular detect lack of coincidences (see Figures 8 and 9) whereas the detections of UE cannot be trusted in this range of parameters. This in particular leads to new insights on these already published data (see Figure 9). Note that on the same data, the symmetric UE method declares statistically significant, 
regions for which the parameter $q$ of MTGAUE needs to be larger than $80 \%$ (see Figure 7). Because of the previous simulation studies, those detections are therefore quite likely to be false positives.

In conclusion, it was already known that the UE method with multiple shift coincidence count (Grün et al., 1999) allowed to avoid information loss compared to the classical UE method based on binned coincidence count (Grün, 1996). In this article, we show that MTGAUE notably extends the properties of this UE method with multiple shift coincidence count thanks to its clear control of the false discovery rate, its robustness, its ability to detect both lack and profusion of coincidences and this for large set of parameters (delays, number of trials, firing rates).

In terms of perspective, it should be possible to extend the use of the delayed coincidence count to the analysis of more than two neurons at a time, as it has already been done for the binned coincidences (see for instance (Grün, 1996; Grün et al., 2010)). This would allow the more direct study of neuronal assemblies. Adaptation of trialshuffling should also be considered. However, we think that pure testing procedures do not give fully satisfying answers and that it would be legitimate to provide an estimation of the dependence notably, through the Hawkes model (Krumin et al., 2010). It is already known that this model can easily deal with more than two neurons (Daley \& Vere-Jones, 2003; Pernice et al., 2011, 2012; Chornoboy et al., 1988). However, it is only in a recent work that we have proposed theoretical statistical methods to deal with several neurons and large delays of interaction through Lasso methods (Hansen et al., 2012). We aim at generalizing those results to non stationary data in time.

\section{Acknowledgments}

We first of all want to thank Alexa Riehle, leader of the laboratory in which the data used in this article were previously collected. We wish also to thank Y. Bouret and F. Picard for fruitful discussions and programming help at several stages of this work. Finally, we thank two anonymous referees for helpful remarks and comments. This research is partly supported by the french Agence Nationale de la Recherche (ANR 2011 BS01 01001 projet Calibration ), by the PEPS BMI 2012-2013 Estimation of dependence graphs for thalamo-cortical neurons and multivariate Hawkes processes and the PEPS BMI 2012-2013 NeuroConf.

\section{References}

Abeles, M. (1982). Quantification, smoothing, and confidence-limits for single-units histograms. Journal of Neuroscience Methods, 5(4), 317-325. 
Abeles, M. (1991). Corticonics. Neural circuits in the cerebral cortex. Cambridge University Press.

Abeles, M., \& Gat, I. (2001). Detecting precise firing sequences in experimental data. Journal of Neuroscience Methods, 107, 141-154.

Aertsen, A.M., Gerstein, G.L., Habib, M.K., \& Palm, G. (1989). Dynamics of neuronal firing correlation: modulation of "effective connectivity". Journal of Neurophysiology, 61(5), 900-917.

Benjamini, Y. (2010). Discovering the false discovery rate. Journal of the Royal Statistical Society, Series B, 72(4), 405-416.

Benjamini, Y. \& Hochberg, Y., (1995). Controlling the false discovery rate: a practical and powerful approach to multiple testing. Journal of the Royal Statistical Society, Series B, 57(1), 289-300.

Benjamini, Y. \& Yekutieli, D., (2001). The control of the false discovery rate in multiple testing under dependency. Annals of Statistics, 29(4), 1165-1188.

Boucsein, C., Nawrot, M.P., Schnepel, P., \& Aertsen, A.M. (2011). Beyond the cortical column: abundance and physiology of horizontal connections imply a strong role for inputs from the surround. Frontiers in Neuroscience, (5), article 32.

Brette, R. (2012). Computing with neural synchrony. PLoS Computational Biology, $8(6), \mathrm{e} 1002561$.

Brown, E., Barbieri, R., Ventura, V., Kass, R., \& Frank, L. (2002). The time rescaling theorem and its application to neural spike train analysis. Neural Computation, 14(2), $325-346$.

Chornoboy, E.S., Schramm, L.P., \& Karr, A.F. (1988). Maximum likelihood identification of neural point process systems., Biological Cybernetics, 59, 265-275.

Daley, D.J., \& Vere-Jones, D. (2003). An introduction to the theory of point processes, vol. I. Springer.

Diesmann, M., Gewaltig, M.O., \& Aertsen, A.M. (1999). Stable propagation of synchronous spiking in cortical neural networks. Nature, 402, 529-533.

Engel, A.K., \& Singer, W. (2001). Temporal binding and the neural correlates of sensory awareness. TRENDS in Cognitive Sciences, 5(1), 16-25. 
Eyherabide, H.G., Rokem, A., Herz, A.V.M., \& Samengo, I. (2009). Bursts generate a non-reducible spike-pattern code. Frontiers in Neuroscience, 3(1), 8-14.

Gerstein, G.L. (2004). Searching for significance in spatio-temporal firing patterns. Acta Neurobiologia Experimentalis, 64, 203-207.

Gerstein, G.L., \& Aertsen, A.M. (1985). Representation of cooperative firing activity among simultaneously recorded neurons. Journal of Neurophysiology, 54(6), 15131528.

Gerstein, G.L., \& Perkel, D.H. (1969). Simultaneous recorded trains of action potentials: analysis and functional interpretation. Science, 164, 828-830.

Goedeke, S., \& Diesmann, M. (2008). The mechanism of synchronization in feedforward neuronal networks. New Journal of Physics, 10, 015007.

Golomb, D., \& Hansel, D. (2000). The number of synaptic inputs and the synchrony of large sparse neuronal networks. Neural Computation, 12(5), 1095-1139.

Grammont, F., \& Riehle, A. (1999). Precise spike synchronization in monkey motor cortex involved in preparation for movement. Experimental Brain Research, 128(12), 118-122.

Grammont, F., \& Riehle, A. (2003). Spike synchronisation and firing rate in a population of motor cortical neurons in relation to movement direction and reaction time. Biological Cybernetics, 88, 360-373.

Grün, S. (1996). Unitary joint-events in multiple-neuron spiking activity: Detection, significance and interpretation. Thun: Verlag Harri Deutsch.

Grün, S. (2009). Data-driven significance estimation for precise spike correlation. Journal of Neurophysiology, 101, 1126-1140.

Grün, S., Diesman, M., \& Aertsen, A.M. (2001a). Unitary events in multiple singleneuron spiking activity: I. Detection and Significance. Neural Computation, 14, 43-80.

Grün, S., Diesman, M., \& Aertsen, A.M. (2001b). Unitary events in multiple singleneuron spiking activity: II. Nonstationary Data. Neural Computation, 14, 81-119.

Grün, S., Diesmann, M., \& Aertsen, A.M. (2010). Unitary Events Analysis. In Analysis of Parallel Spike Trains, Grün, S., \& Rotter, S., Springer Series in Computational Neuroscience. 
Grün, S., Diesmann, M., Grammont, F., Riehle, A., \& Aertsen, A.M. (1999). Detecting unitary events without discretization of time. Journal of Neuroscience Methods, 93, $67-79$.

Grün, S., Riehle, A., \& Diesmann, M. (1999). Effect of cross-trial nonstationarity on joint-spike events. Biological Cybernetics, 88, 335-351.

Gütig, R., Aertsen, A.M., \& Rotter, S. (2001). Statistical Significance of Coincident Spikes: Count-Based Versus Rate-Based Statistics. Neural Computation, 14, 121153.

Hansen, N.R., Reynaud-Bouret, P., \& Rivoirard, V. (2012). Lasso and probabilistic inequalities for multivariate point processes. http://arxiv.org/abs/1208.0570, in revision.

Hebb, D. O. (1949). Organization of behavior; a neuropsychological theory. New York: John Wiley \& Sons.

Heinzle, J., König, P., \& Salazar R.F. (2007). Modulation of synchrony without changes in firing rate. Cognitive Neurodynamics, 1, 225-235.

Hochberg, Y., \& Tamhame, A. (1987). Multiple comparison procedures. New York: Wiley.

Hogg, R.V., \& Tanis, E.A. (2009). Probability and statistical inference. Pearson.

Holm, S. (1979). A simple sequentially rejection multiple test procedure. Scandinavian Journal of Statistics, 6(2), 65-70.

König, P., Engel, A.K., \& Singer, W. (1996). Integrator or coincidence detector? The role of the cortical neuron revisited. Trends in Neurosciences, 19(4), 130-137.

Konishi, M., Takahashi, T.T., Wagner, H., Sullivan, W.E., \& Carr, C.E. (1988). Neurophysiological and anatomical substrates of sound localization in the owl. In Auditory Function, Edelman, G.M., Gall, W.E. and Cowan, W.M., Wiley, New-York.

Krumin, M., Reutsky, I., \& Shoham, S. (2010). Correlation-based analysis and generation of multiple spike trains using Hawkes models with an exogenous input. Frontiers in Computational Neuroscience, 4, article 147.

Kumar, A., Rotter, S., \& Aertsen, A.M. (2010). Spiking activity propagation in neuronal networks: reconciling different perspectives on neural coding. Nature Reviews Neuroscience, 11, 615-627. 
Lestienne, R. (1996). Determination of the precision of spike timing in the visual cortex of anaesthetised cats. Biological Cybernetics, 74, 55-61.

Lestienne, R. (2001). Spike timing, synchronization and information processing on the sensory side of the central nervous system. Progress in Neurobiology, 65, 545-591.

Louis, S., Borgelt, C., \& Grün, S. (2010). Generation and Selection of Surrogate Methods for Correlation Analysis. In Analysis of Parallel Spike Trains, Grün, S., \& Rotter, S., Springer Series in Computational Neuroscience.

Mainen, Z.F., \& Sejnowski, T.J. (1995). Reliability of spike timing in neocortical neurons. Science, 268, 1503-1506.

Maimon, G., \& Assad, J.A. (2009). Beyond Poisson: increased spike-time regularity across primate parietal cortex. Neuron, 62(3), 426-440.

Maldonado, P.E., Babul, C., Singer, W., Rodriguez, E., Berger, D., \& Grün, S. (2008). Synchronization of neuronal response in primary visual cortex of monkeys viewing natural images. Journal of Neurophysiology, 100, 1523-1532.

Maldonado, P.E., Friedman-Hill, S., \& Gray, C.M. (2000). Dynamics of striate cortical activity in the alert macaque: II. Fast time scale synchronization. Cerebral Cortex, 10, 1117-1131.

Masuda, N., \& Aihara, K. (2007). Dual coding hypotheses for neural information representation. Mathematical Biosciences, 207, 312-321.

Ogata, Y. (1981). On Lewis Simulation Method for Point Processes. Journal of the American Statistical Association, 83(401), 9-27.

Ogata, Y. (1988). Statistical models for earthquakes occurrences and residual analysis for point processes. IEEE Transactions on Information Theory, 27(1), 23-31.

Palm, G. (1990). Cell assemblies as a guideline for brain research. Concepts in Neuroscience, $1,133-148$.

Pazienti, A., Maldonado, P.E., Diesmann, M., \& Grün, S. (2008). Effectiveness of systematic spike dithering depends on the precision of cortical synchronization. Brain Research, 1225, 39-46.

Perkel, D.H., Gernstein, G.L., \& Moore, G.P. (1967). Neuronal spike trains and stochastic point processes. Biophysical Journal, 7, 419-440. 
Pernice, V., Staude, B., Cardanobile, S., \& Rotter, S. (2011). How structure determines correlations in neuronal networks. PLoS Computational Biology, 7, e1002059.

Pernice, V., Staude, B., Cardanobile, S., \& Rotter, S. Recurrent interactions in spiking networks with arbitrary topology. Physical review E, Statistical, nonlinear, and soft matter physics, 85, 031916.

Pipa, G., Diesmann, M., \& Grün, S. (2003). Significance of joint-spike events based on trial-shuffling by efficient combinatorial methods. Complexity, 8(4), 1-8.

Pipa, G., \& Grün, S. (2003). Non-parametric significance estimation of joint-spike events by shuffling and resampling. Neurocomputing, 52-54, 31-37.

Pipa, G., Grün, S., \& van Vreeswijk, C. (2013). Impact of Spike Train Autostructure on Probability Distribution of Joint Spike Events. Neural Computation, 25, 1123-1163.

Prescott, S.A., Ratté, S., De Koninck, Y., \& Sejnowski, T.J. (2008). Pyramidal neurons switch from integrators in vitro to resonators under in vivo-like conditions. Journal of Neurophysiology, 100, 3030-3042.

Reimer, I.C.G., Staude, B., Ehm, W., \& Rotter, S. (2012). Modeling and analyzing higher-order correlations in non-Poissonian spike trains. Journal of Neuroscience Methods, 208, 18-33.

Reynaud-Bouret, P., Tuleau-Malot, C., Rivoirard, V., \& Grammont, F. (2013). Spike trains as (in)homogeneous Poisson processes or Hawkes processes: non-parametric adaptive estimation and goodness-of-fit tests. http://hal.archives-ouvertes.fr/hal00789127, submitted.

Riehle, A., Grammont, F., Diesmann, M. \& Grün, S. (2000). Dynamical changes and temporal precision of synchronised spiking activity in monkey motor cortex during movement preparation. Journal of Physiology, 94, 569-582.

Riehle, A., Grammont, F., \& MacKay, A. (2006). Cancellation of a planned movement in monkey motor cortex. Neuroreport, 17(3), 281-285.

Riehle, A., Grün, S., Diesmann, M., \& Aertsen, A.M. (1997). Spike synchronization and rate modulation differentially involved in motor cortical function. Science, 278, 1950-1953.

Roy, A., Steinmetz, P.N., \& Niebur, E. (2000). Rate Limitations of Unitary Event Analysis. Neural Computation, 12(9), 2063-2082. 
Rudolph, M., \& Destexhe, A. (2003). Tuning neocortical pyramidal neurons between integrators and coincidence detectors. Journal of Computational Neuroscience, 14, 239-251.

Sakurai, Y. (1999). How do cell assemblies encode information in the brain? Neuroscience and Biobehavioral Reviews, 23, 785-796.

Shinomoto, S. (2010). Estimating the Firing Rate. In Analysis of Parallel Spike Trains, Grün, S., \& Rotter, S., Springer Series in Computational Neuroscience.

Singer, W. (1993). Synchronization of cortical activity and its putative role in information processing and learning. Annual Review of Physiology, 55, 349-374.

Singer, W. (1999). Neuronal synchrony: a versatile code for the definition of relations? Neuron, 24, 49-65.

Singer, W., \& Gray, C.M. (1995). Visual feature integration and the temporal correlation hypothesis. Annual Review of Neuroscience, 18, 555-586.

Softky, W.R., \& Koch, C. (1993). The highly irregular firing of cortical cells is inconsistent with temporal integration of random EPSPs. Journal of Neuroscience, 13(1), 334-350.

Staude, B., Rotter, S., \& Grün, S. (2010). CuBIC: cumulant based inference of higherorder correlations in massively parallel spike trains. Journal of Computational Neuroscience, 29, 327-350.

Super, H., van der Togt, C., Spekreijse, H., Lamme, V.A. (2003). Internal state of monkey primary visual cortex (V1) predicts figure-ground perception. Journal of Neuroscience, 23(8), 3407-3414.

Tiesinga, P.H.E, \& Sejnowski, T.J. (2004). Rapid temporal modulation of synchrony by competition in cortical interneuron networks. Neural Computation, 16, 251-275.

Vaadia, E., Haalman, I., Abeles, M., Bergman, H., Prut, Y., Slovin, H., \& Aertsen, A. (1995). Dynamics of neuronal interactions in monkey cortex in relation to behavioural events. Nature, 373, 515-518.

Ventura, V. (2010). Bootstrap Tests of Hypotheses. In Analysis of Parallel Spike Trains, Grün, S., \& Rotter, S., Springer Series in Computational Neuroscience.

Victor, J.D., \& Purpura, K.P. (1996). Nature and precision of temporal coding in visual cortex: a metric-space analysis. Journal of Neurophysiology, 76(2), 1310-1326. 
Von der Malsburg, C. (1981). The correlation theory of brain function. Internal Report. Göttingen: Max-Planck-Institute for Biophysical chemistry, Dept. Neurobiology. 